Supporting Information Section

(a)
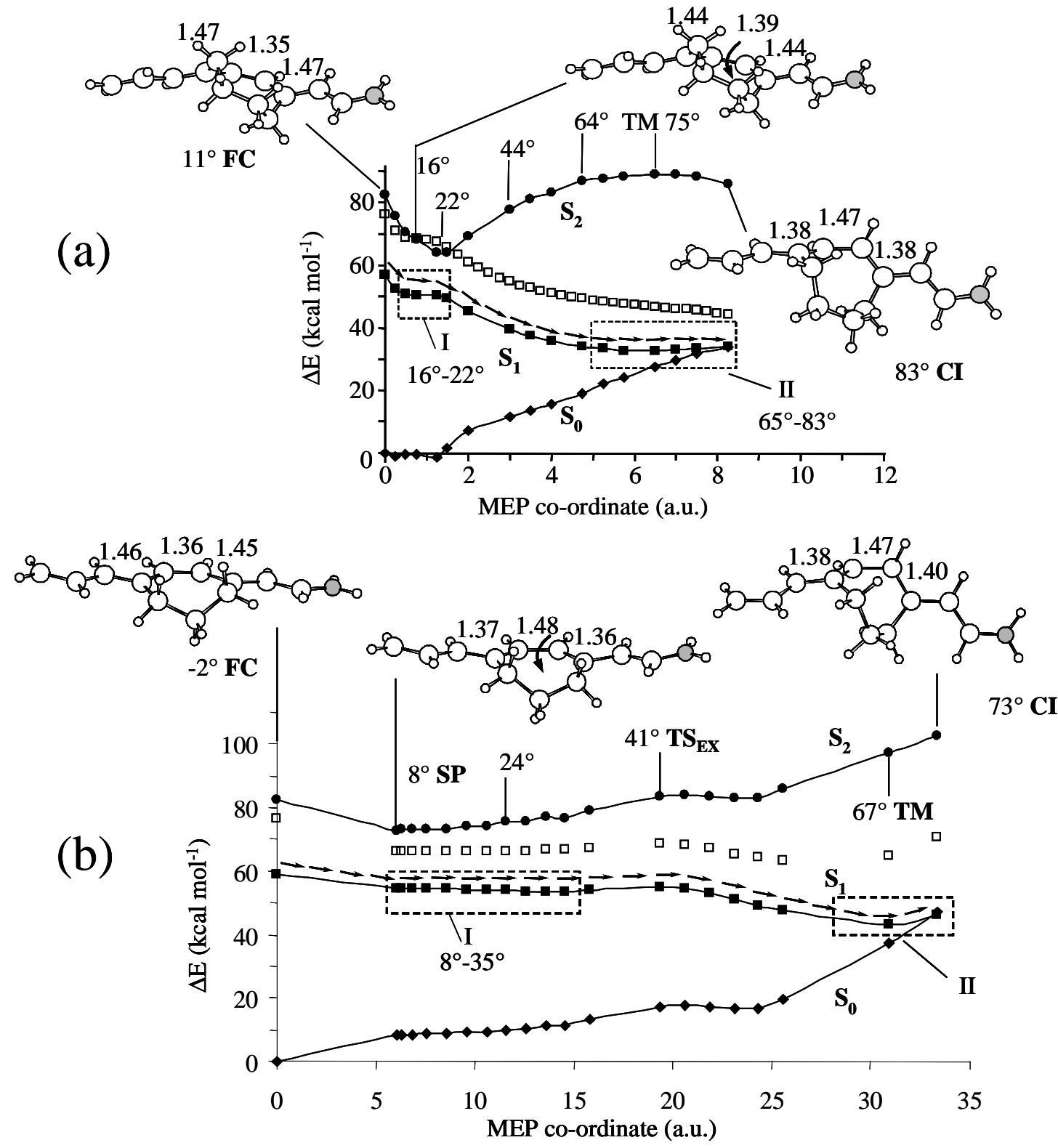

Figure 6S

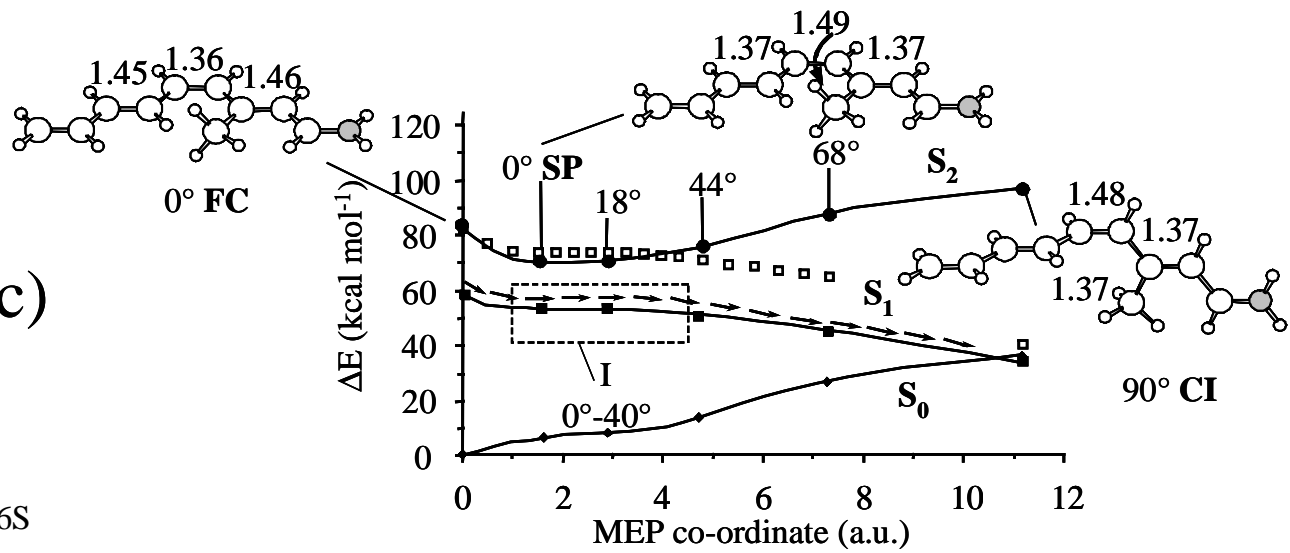


Caption

Figure 6S. Energy profiles along the (a) $\mathrm{S}_{1}$ 4-cis $\rightarrow$ all-trans (11-cis $\rightarrow$ all-trans in PSB11.8) photoisomerization coordinate of $\mathbf{3}$, (b) $\mathrm{S}_{1}$ 4-cis $\rightarrow$ all-trans (11-cis $\rightarrow$ all-trans in PSB11.7) photoisomerization coordinate of 2 and (c) $\mathrm{S}_{1}$ 4-cis $\rightarrow$ all-trans (11-cis $\rightarrow$ all-trans in PSB11) photoisomerization coordinate of $\mathbf{1}$ (data from ref. ${ }^{10}$ ). Full circles, full squares and full diamonds indicate the $S_{2}, S_{1}$ and $S_{0}$ CASPT2 energies. Open squares show the $S_{1}$ CASSCF energies (i.e. before PT2 correction). The structures (geometrical parameters in $\AA$ and degrees) document the progression of the molecular structure along the co-ordinate. FC is the Franck-Condon structure, SP corresponds to the relaxed planar species, $\mathbf{T S}_{\mathbf{E X}}$ is a transition state, $\mathbf{T M}$ represents a relaxed twisted minimum and $\mathbf{C I}$ is the ca. $90^{\circ}$ twisted $\mathrm{S}_{1} / \mathrm{S}_{0}$ conical intersection decay channel. 


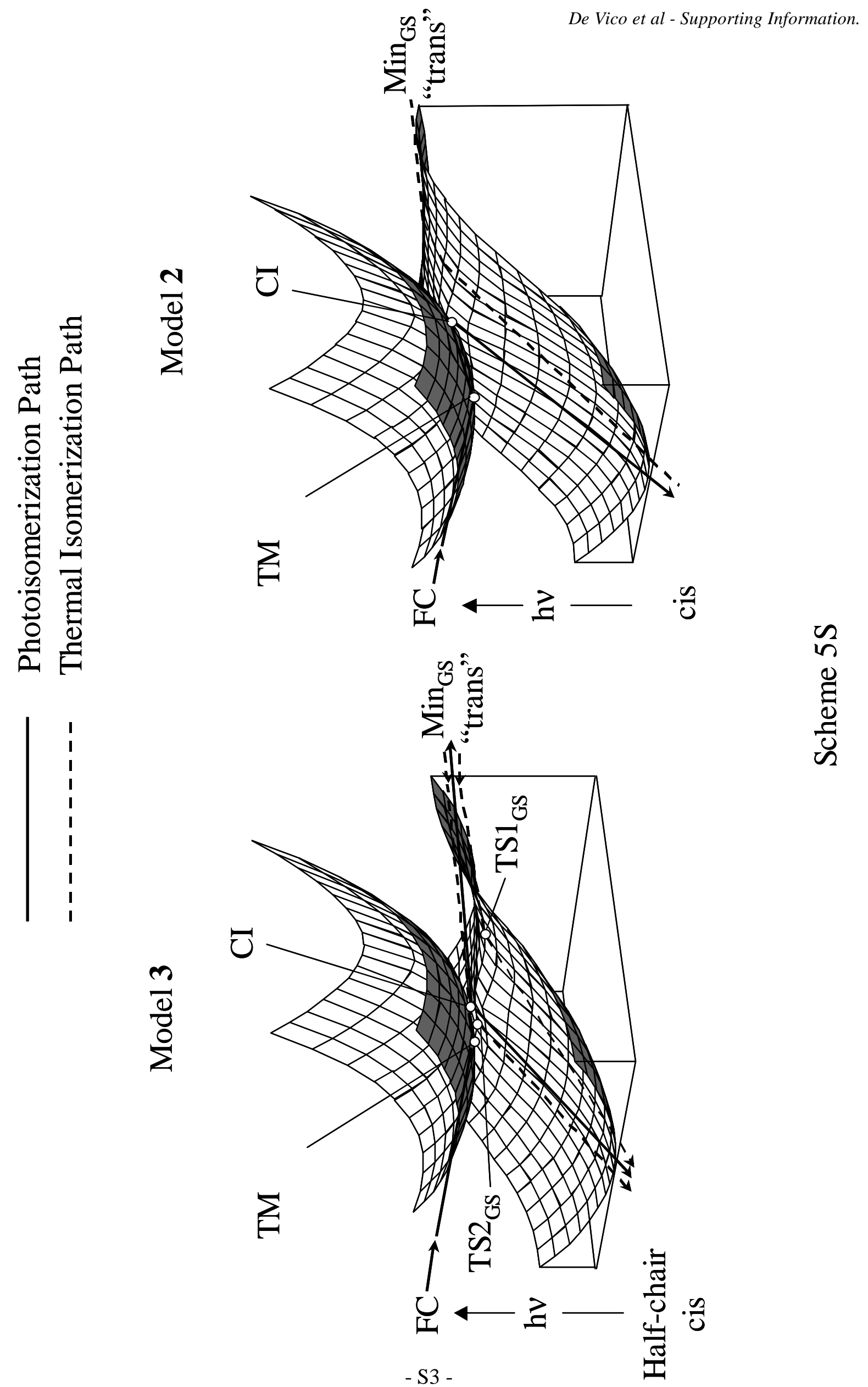



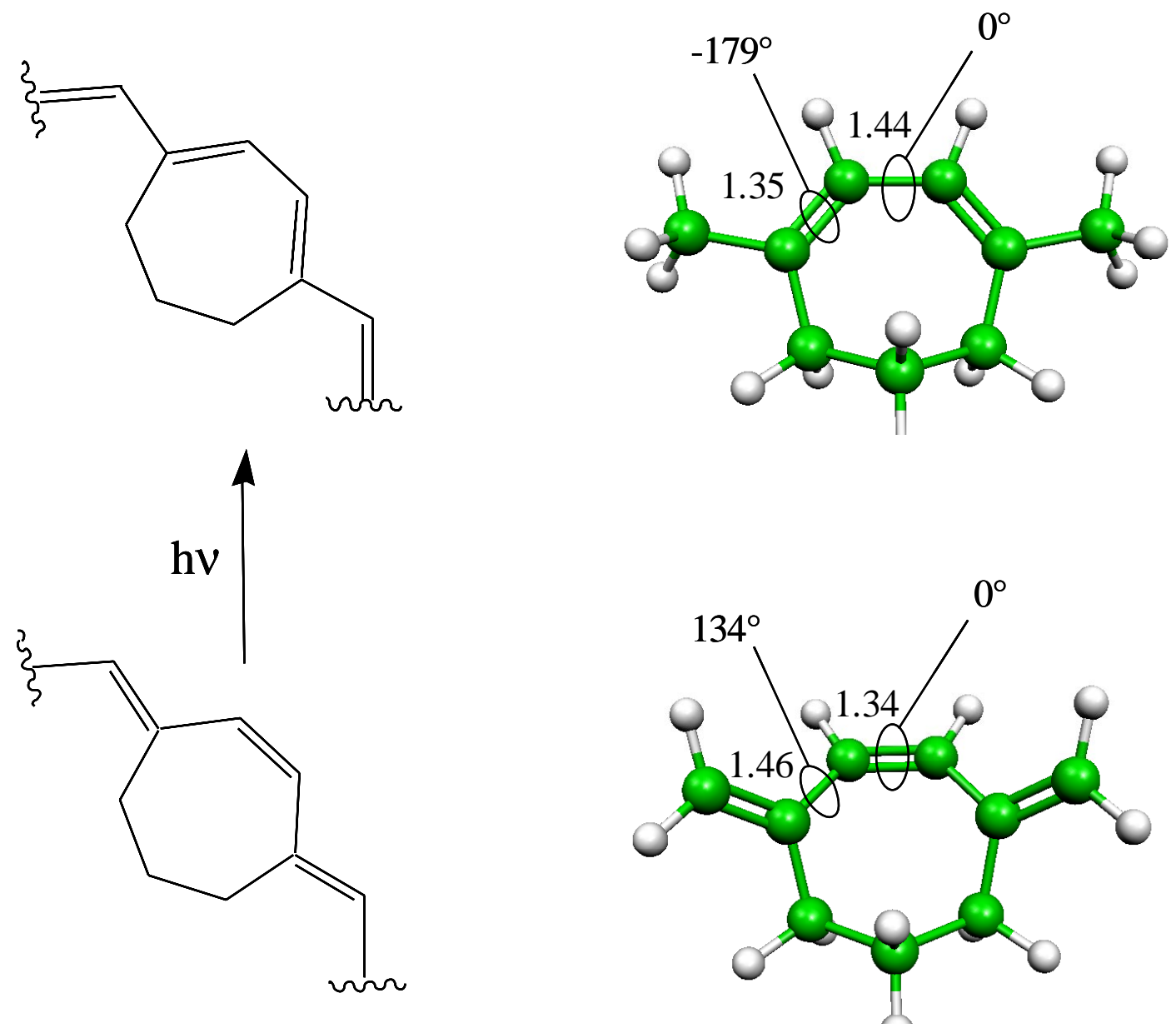

Scheme 6S 
Table I. CASSCF/6-31G* Absolute, CASPT2/6-31G* Absolute and Relative Energies along with Wave Function Reference Weights of the Structures Discussed in the Text

\begin{tabular}{|c|c|c|c|c|c|}
\hline Structure & State & $\begin{array}{l}\text { CASSCF energy } \\
\text { (hartree) }\end{array}$ & $\begin{array}{l}\text { CASPT2 energy } \\
\text { (hartree) }^{\mathrm{a}}\end{array}$ & $\begin{array}{l}\text { reference } \\
\text { weight }^{\mathrm{a}}\end{array}$ & $\begin{array}{l}\text { CASPT2 level relative } \\
\text { energy }\left(\mathrm{kcal} \mathrm{mol}^{-1}\right)^{\mathrm{b}}\end{array}$ \\
\hline \multicolumn{6}{|c|}{ 2-(4-allylidene-cyclohepta-2-enylidene)-ethyliminium cation model 2} \\
\hline \multirow[t]{3}{*}{ FC } & $\mathrm{S}_{0}$ & -518.0385 & -519.6694 & 0.65 & 0.0 \\
\hline & $\mathrm{S}_{1}$ & & -519.5756 & 0.64 & 58.9 \\
\hline & $\mathrm{S}_{2}$ & & -519.5376 & 0.63 & 82.7 \\
\hline \multirow[t]{3}{*}{ SP } & $\mathrm{S}_{0}$ & -518.0022 & -519.6560 & 0.65 & 8.4 \\
\hline & $\mathrm{S}_{1}$ & -517.9215 & -519.5822 & 0.64 & 54.7 \\
\hline & $\mathrm{S}_{2}$ & & $-519.5533^{\mathrm{c}}$ & $0.64^{\mathrm{c}}$ & 72.9 \\
\hline \multirow[t]{3}{*}{$\mathbf{T S}_{\mathrm{EX}}$} & $\mathrm{S}_{0}$ & -517.9846 & -519.6421 & 0.65 & 17.1 \\
\hline & $\mathrm{S}_{1}$ & -517.9187 & -519.5817 & 0.64 & 55.0 \\
\hline & $\mathrm{S}_{2}$ & & $-519.5361^{c}$ & $0.64^{\mathrm{c}}$ & 83.6 \\
\hline \multirow[t]{3}{*}{ TM } & $\mathrm{S}_{0}$ & -517.9486 & -519.6096 & 0.64 & 37.6 \\
\hline & $S_{1}$ & -517.9319 & -519.6002 & 0.64 & 43.4 \\
\hline & $\mathrm{S}_{2}$ & & $-519.5144^{c}$ & $0.64^{\mathrm{c}}$ & 97.3 \\
\hline \multirow[t]{3}{*}{ CI } & $\mathrm{S}_{0}$ & -517.9294 & -519.5955 & 0.64 & 46.4 \\
\hline & $\mathrm{S}_{1}$ & -517.9294 & -519.5943 & 0.64 & 47.1 \\
\hline & $\mathrm{S}_{2}$ & & -519.5062 & 0.58 & 102.4 \\
\hline \multirow[t]{3}{*}{$\mathbf{M I N}_{\mathrm{GS}}$} & $\mathrm{S}_{0}$ & -517.9612 & -519.5896 & 0.64 & 50.1 \\
\hline & $\mathrm{S}_{1}$ & & -519.4974 & 0.63 & 107.9 \\
\hline & $\mathrm{S}_{2}$ & & $-519.4702^{\mathrm{c}}$ & $0.63^{\mathrm{c}}$ & 125.0 \\
\hline \multicolumn{6}{|c|}{ 2-(4-allylidene-cycloocta-2-enylidene)-ethyliminium cation model $\mathbf{3}$} \\
\hline \multirow[t]{3}{*}{ Half-chair (FC) } & $\mathrm{S}_{0}$ & -557.0472 & -558.8322 & 0.63 & 0.0 \\
\hline & $\mathrm{S}_{1}$ & -556.9255 & -558.7415 & 0.62 & 56.9 \\
\hline & $\mathrm{S}_{2}$ & & -558.6991 & $0.63^{\mathrm{c}}$ & 82.5 \\
\hline \multirow[t]{3}{*}{ Boat-1 } & $\mathrm{S}_{0}$ & -557.0648 & -558.8334 & 0.63 & -0.8 \\
\hline & $\mathrm{S}_{1}$ & & -558.7387 & 0.61 & 58.7 \\
\hline & $\mathrm{S}_{2}$ & & $-558.6971^{\mathrm{c}}$ & $0.63^{\mathrm{c}}$ & 84.8 \\
\hline \multirow[t]{3}{*}{ Boat-2 } & $\mathrm{S}_{0}$ & -557.0644 & -558.8319 & 0.63 & 0.2 \\
\hline & $\mathrm{S}_{1}$ & & -558.7423 & 0.62 & 56.4 \\
\hline & $\mathrm{S}_{2}$ & & -558.6872 & 0.55 & 91.0 \\
\hline \multirow[t]{3}{*}{ CI } & $\mathrm{S}_{0}$ & -556.9778 & -558.7785 & 0.63 & 33.6 \\
\hline & $S_{1}$ & -556.9766 & -558.7777 & 0.62 & 34.2 \\
\hline & $\mathrm{S}_{2}$ & & $-558.6954^{\mathrm{c}}$ & $0.61^{\mathrm{c}}$ & 85.8 \\
\hline \multirow[t]{3}{*}{$\mathbf{M I N}_{\mathrm{GS}}$} & $\mathrm{S}_{0}$ & -556.0283 & -558.7919 & 0.62 & 25.3 \\
\hline & $\mathrm{S}_{1}$ & & -558.6910 & 0.60 & 88.6 \\
\hline & $\mathrm{S}_{2}$ & & $-558.6632^{c}$ & $0.63^{c}$ & 106.0 \\
\hline \multirow[t]{3}{*}{$\mathbf{T S 1}_{\mathrm{GS}}$} & $\mathrm{S}_{0}$ & -556.9897 & -558.7894 & 0.62 & 26.8 \\
\hline & $\mathrm{S}_{1}$ & & -558.7442 & 0.62 & 55.2 \\
\hline & $\mathrm{S}_{2}$ & & $-558.6952^{c}$ & $0.60^{\mathrm{c}}$ & 86.0 \\
\hline \multirow[t]{3}{*}{$\mathbf{T S} 2_{\mathrm{GS}}$} & $\mathrm{S}_{0}$ & -556.9937 & -558.7805 & 0.62 & 32.4 \\
\hline & $\mathrm{S}_{1}$ & & -558.7578 & 0.62 & 46.7 \\
\hline & $\mathrm{S}_{2}$ & & $-558.6869^{c}$ & $0.63^{\mathrm{c}}$ & 91.2 \\
\hline \multirow[t]{3}{*}{$\mathbf{M I N}_{\mathrm{EX}}$} & $\mathrm{S}_{0}$ & -557.0163 & -558.8109 & 0.62 & 13.4 \\
\hline & $\mathrm{S}_{1}$ & -556.9417 & -558.7518 & 0.62 & 50.5 \\
\hline & $\mathrm{S}_{2}$ & & -558.6845 & 0.56 & 92.7 \\
\hline \multirow[t]{3}{*}{$\mathbf{T S}_{\mathrm{EX}}$} & $\mathrm{S}_{0}$ & -557.0028 & -558.8036 & 0.63 & 17.5 \\
\hline & $\mathrm{S}_{1}$ & -556.9225 & -558.7396 & 0.61 & 58.2 \\
\hline & $\mathrm{S}_{2}$ & & $-558.7074^{\mathrm{c}}$ & $0.63^{\mathrm{c}}$ & 78.1 \\
\hline \multirow[t]{3}{*}{$\mathrm{CI}^{\prime}$} & $\mathrm{S}_{0}$ & -556.9545 & -558.7569 & 0.62 & 47.2 \\
\hline & $\mathrm{S}_{1}$ & -556.9545 & -558.7578 & 0.62 & 46.7 \\
\hline & $\mathrm{S}_{2}$ & & $-558.7000^{c}$ & $0.56^{\mathrm{c}}$ & 82.9 \\
\hline
\end{tabular}

\footnotetext{
${ }^{\text {a }}$ A three roots $\left(\mathrm{S}_{0}, \mathrm{~S}_{1}, \mathrm{~S}_{2}\right)$ state average $(0.33,0.33,0.33)$ CASSCF wave function (energies not shown) was used as zeroth order wave function for the CASPT2 results quoted (energies and weights)

${ }^{\mathrm{b}}$ Energies are relative to the $\mathrm{S}_{0}$ state energy value of $\mathbf{F C}$ of each model

${ }^{\mathrm{c}}$ CASPT2 energy obtained applying a level shift procedure, with level shift equal to 0.1 .
} 
Table II. CASSCF/6-31G(d,p) Absolute, CASPT2/6-31G(d,p) Absolute Energies along with Wave Function Reference Weights of the Conical Intersections Discussed in the Text. Energy difference between $S_{0}$ and $S_{1}$ CASPT2 energies is also given.

\begin{tabular}{|c|c|c|c|c|c|}
\hline Structure & State & $\begin{array}{c}\text { CASSCF reference } \\
\text { energy (hartree) }\end{array}$ & $\begin{array}{c}\text { CASPT2 energy } \\
\text { (hartree) }\end{array}$ & $\begin{array}{l}\text { reference } \\
\text { weight }\end{array}$ & $\begin{array}{l}\text { CASPT2 } \mathrm{S}_{0} / \mathrm{S}_{1} \text { energy } \\
\text { difference }\left(\mathrm{kcal} \mathrm{mol}^{-1}\right)\end{array}$ \\
\hline \multicolumn{6}{|c|}{ 2-(4-allylidene-cyclohepta-2-enylidene)-ethyliminium cation model 2} \\
\hline \multirow[t]{4}{*}{ CI } & $\mathrm{S}_{0}$ & -517.9703 & -519.7278 & 0.64 & \multirow[t]{3}{*}{0.7} \\
\hline & $\mathrm{S}_{1}$ & -517.9450 & -519.7289 & 0.63 & \\
\hline & $\mathrm{S}_{2}$ & -517.8759 & $-519.6393^{\mathrm{a}}$ & $0.63^{\mathrm{a}}$ & \\
\hline & \multicolumn{5}{|c|}{ 2-(4-allylidene-cycloocta-2-enylidene)-ethyliminium cation model 3} \\
\hline \multirow[t]{3}{*}{ CI } & $\mathrm{S}_{0}$ & -557.0204 & -558.9272 & 0.62 & \multirow[t]{3}{*}{0.1} \\
\hline & $\mathrm{S}_{1}$ & -556.9957 & -558.9271 & 0.61 & \\
\hline & $\mathrm{S}_{2}$ & -556.9233 & $-558.8363^{\mathrm{a}}$ & $0.61^{\mathrm{a}}$ & \\
\hline
\end{tabular}

${ }^{\text {a }}$ CASPT2 energy obtained applying a level shift procedure, with level shift equal to 0.1 . 
Table III. Cartesian coordinates of the structures discussed in the text. All data are in angstrom.

$$
\text { 2-(4-allylidene-cyclohepta-2-enylidene)-ethyliminium cation model } 2
$$

FC

$\begin{array}{llll}\text { C } & -0.398645 & 1.276158 & -0.328383\end{array}$

$\begin{array}{llll}\text { C } & 0.951985 & 1.286301 & -0.155111\end{array}$

$\begin{array}{llll}\text { C } & -1.465098 & 0.345470 & 0.026792\end{array}$

$\begin{array}{llll}\text { C } & 1.836182 & 0.342221 & 0.503962\end{array}$

$\begin{array}{llll}\text { C } & 3.179002 & 0.621163 & 0.431159\end{array}$

$\begin{array}{llll}\text { C } & 1.272254 & -0.842696 & 1.249237\end{array}$

$\begin{array}{llll}\text { C } & 4.209006 & -0.153851 & 1.022691\end{array}$

$\begin{array}{llll}\text { C } & -1.218650 & -1.017558 & 0.648450\end{array}$

$\begin{array}{llll}\text { C } & -2.733625 & 0.779168 & -0.225391\end{array}$

$\begin{array}{llll}\text { C } & -3.979078 & 0.076354 & 0.060336\end{array}$

$\begin{array}{llll}\mathrm{N} & 5.464361 & 0.158200 & 0.937951\end{array}$

$\begin{array}{llll}\text { C } & -5.186331 & 0.594986 & -0.237153\end{array}$

$\begin{array}{llll}\text { C } & 0.180505 & -1.603477 & 0.481959\end{array}$

$\mathrm{H} \quad-0.761883 \quad 2.146547 \quad-0.845824$

$\begin{array}{llll}\mathrm{H} & 1.453975 & 2.140587 & -0.573841\end{array}$

H $\quad 3.496546 \quad 1.497614 \quad-0.106494$

$\begin{array}{llll}\mathrm{H} & 2.058760 & -1.539089 & 1.506660\end{array}$

$\begin{array}{llll}\mathrm{H} & 0.867452 & -0.478802 & 2.190217\end{array}$

$\mathrm{H} \quad 3.997812 \quad-1.046441 \quad 1.577301$

$\begin{array}{llll}\mathrm{H} & -1.917002 & -1.721581 & 0.213809\end{array}$

$\begin{array}{llll}\mathrm{H} & -1.451993 & -0.976859 & 1.710036\end{array}$

$\mathrm{H} \quad-2.854581 \quad 1.752238 \quad-0.670683$

$\begin{array}{llll}\mathrm{H} & -3.932668 & -0.886948 & 0.534440\end{array}$

$\begin{array}{llll}\mathrm{H} & 6.174835 & -0.404281 & 1.362198\end{array}$

$\begin{array}{llll}\mathrm{H} & 5.773604 & 0.974007 & 0.444733\end{array}$

$\mathrm{H} \quad-6.093150 \quad 0.064471 \quad-0.015514$

$\mathrm{H} \quad-5.290388 \quad 1.557458 \quad-0.706223$

$\begin{array}{llll}\mathrm{H} & 0.156239 & -2.616936 & 0.866574\end{array}$

$\begin{array}{llll}\mathrm{H} & 0.444013 & -1.680868 & -0.568515\end{array}$

\section{SP $8^{\circ}$}

$\begin{array}{lrrr}\mathrm{C} & -0.474459 & 1.178678 & -0.471478 \\ \mathrm{C} & 0.995059 & 1.160606 & -0.267567 \\ \mathrm{C} & -1.455129 & 0.353017 & 0.002204 \\ \mathrm{C} & 1.810919 & 0.249924 & 0.323117 \\ \mathrm{C} & 3.231494 & 0.633545 & 0.443041 \\ \mathrm{C} & 1.396617 & -1.089295 & 0.880965 \\ \mathrm{C} & 4.195399 & -0.055387 & 1.155316 \\ \mathrm{C} & -1.183758 & -0.898256 & 0.809209 \\ \mathrm{C} & -2.811670 & 0.758487 & -0.290103 \\ \mathrm{C} & -3.997746 & 0.162595 & 0.167628 \\ \mathrm{~N} & 5.464983 & 0.345042 & 1.265025 \\ \mathrm{C} & -5.236415 & 0.663656 & -0.171777 \\ \mathrm{C} & 0.071697 & -1.664926 & 0.385995 \\ \mathrm{H} & -0.797826 & 2.024947 & -1.047651\end{array}$




$\begin{array}{rrrr}\mathrm{H} & 1.465188 & 2.056280 & -0.630737 \\ \mathrm{H} & 3.530678 & 1.551459 & -0.036395 \\ \mathrm{H} & 2.169724 & -1.808882 & 0.632493 \\ \mathrm{H} & 1.381924 & -1.025208 & 1.967110 \\ \mathrm{H} & 3.966279 & -0.963008 & 1.674778 \\ \mathrm{H} & -2.024032 & -1.570967 & 0.702827 \\ \mathrm{H} & -1.128563 & -0.645067 & 1.865357 \\ \mathrm{H} & -2.923594 & 1.631988 & -0.910726 \\ \mathrm{H} & -3.951367 & -0.698793 & 0.807661 \\ \mathrm{H} & 6.129628 & -0.183148 & 1.786592 \\ \mathrm{H} & 5.796258 & 1.176253 & 0.823240 \\ \mathrm{H} & -6.139729 & 0.207205 & 0.187724 \\ \mathrm{H} & -5.339820 & 1.526304 & -0.806032 \\ \mathrm{H} & -0.003336 & -2.671239 & 0.782592 \\ \mathrm{H} & 0.095034 & -1.762257 & -0.695028\end{array}$

MEP 24

$\begin{array}{lrrr} & & & \\ \mathrm{C} & -0.478787 & 1.269978 & -0.480282 \\ \mathrm{C} & 0.981516 & 1.273346 & -0.204251 \\ \mathrm{C} & -1.446642 & 0.487185 & 0.083843 \\ \mathrm{C} & 1.789550 & 0.302909 & 0.294448 \\ \mathrm{C} & 1.205930 & 0.663683 & 0.502427 \\ \mathrm{C} & 4.152463 & -0.146726 & 1.102558 \\ \mathrm{C} & -1.091668 & -0.619013 & 1.052405 \\ \mathrm{C} & -2.805506 & 0.740311 & -0.322026 \\ \mathrm{C} & -3.966378 & 0.084125 & 0.121820 \\ \mathrm{~N} & 5.421411 & 0.215373 & 1.304354 \\ \mathrm{C} & -5.215019 & 0.424781 & -0.346233 \\ \mathrm{C} & -0.034461 & -1.565513 & 0.470307 \\ \mathrm{H} & -0.788361 & 2.015363 & -1.189388 \\ \mathrm{H} & 1.445012 & 2.214029 & -0.443444 \\ \mathrm{H} & 3.518067 & 1.645386 & 0.185524 \\ \mathrm{H} & 2.059463 & -1.789293 & 0.148132 \\ \mathrm{H} & 1.644962 & -1.224530 & 1.737151 \\ \mathrm{H} & 3.910324 & -1.130614 & 1.447019 \\ \mathrm{H} & -1.969786 & -1.192688 & 1.304920 \\ \mathrm{H} & -0.738382 & -0.186078 & 1.983518 \\ \mathrm{H} & -2.946656 & 1.526733 & -1.044860 \\ \mathrm{H} & -3.891395 & -0.699581 & 0.851780 \\ \mathrm{H} & 6.070593 & -0.400720 & 1.742965 \\ \mathrm{H} & 5.762592 & 1.111275 & 1.026888 \\ \mathrm{H} & -6.097402 & -0.077200 & 0.003923 \\ \mathrm{H} & -5.348280 & 1.206538 & -1.072648 \\ \mathrm{H} & -0.124849 & -2.537189 & 0.943874 \\ \mathrm{H} & -0.229812 & -1.720885 & -0.585274 \\ & & & \\ & & & \end{array}$

$\mathbf{T S}_{\mathbf{E X}} 41^{\circ}$

$\begin{array}{llll}\text { C } & -0.444909 & 1.372257 & -0.469694 \\ \text { C } & 0.991391 & 1.382288 & -0.082477\end{array}$

$\begin{array}{llll}\text { C } & 0.991391 & 1.382288 & -0.082477\end{array}$ 


$\begin{array}{rrrr}\mathrm{C} & -1.411293 & 0.653327 & 0.165338 \\ \mathrm{C} & 1.754280 & 0.326392 & 0.305168 \\ \mathrm{C} & 3.148327 & 0.637062 & 0.633079 \\ \mathrm{C} & 1.291142 & -1.120588 & 0.350233 \\ \mathrm{C} & 4.134708 & -0.250654 & 1.016965 \\ \mathrm{C} & -1.012885 & -0.273972 & 1.291532 \\ \mathrm{C} & -2.754514 & 0.736795 & -0.353331 \\ \mathrm{C} & -3.872613 & -0.009908 & 0.048430 \\ \mathrm{~N} & 5.385849 & 0.116431 & 1.302973 \\ \mathrm{C} & -5.102385 & 0.146508 & -0.553116 \\ \mathrm{C} & -0.171616 & -1.446653 & 0.770762 \\ \mathrm{~N} & -0.710631 & 2.001469 & -1.299406 \\ \mathrm{~N} & 1.452420 & 2.353950 & -0.115715 \\ \mathrm{~N} & 3.431899 & 1.674848 & 0.563725 \\ \mathrm{~N} & 1.483489 & -1.550154 & -0.627963 \\ \mathrm{~N} & 1.937186 & -1.654168 & 1.032460 \\ \mathrm{~N} & 3.950945 & -1.301525 & 1.103104 \\ \mathrm{~N} & -1.883651 & -0.664224 & 1.799289 \\ \mathrm{~N} & -0.455557 & 0.287655 & 2.034153 \\ \mathrm{~N} & -2.905799 & 1.437656 & -1.157359 \\ \mathrm{~N} & -3.776310 & -0.730077 & 0.839558 \\ \mathrm{~N} & 6.072807 & -0.555027 & 1.567604 \\ \mathrm{~N} & 5.679942 & 1.069016 & 1.257638 \\ \mathrm{~N} & -5.951674 & -0.433667 & -0.245224 \\ \mathrm{~N} & -5.252244 & 0.852665 & -1.350205 \\ \mathrm{~N} & -0.131809 & -2.206969 & 1.542015 \\ \mathrm{~N} & -0.690142 & -1.897340 & -0.067559\end{array}$

MEP $53^{\circ}$

$\begin{array}{lrrr}\mathrm{C} & -0.425431 & 1.394350 & -0.519486 \\ \mathrm{C} & 0.990979 & 1.481342 & -0.059131 \\ \mathrm{C} & -1.373525 & 0.672701 & 0.147324 \\ \mathrm{C} & 1.762636 & 0.387008 & 0.197556 \\ \mathrm{C} & 3.116828 & 0.609895 & 0.649092 \\ \mathrm{C} & 1.141352 & -0.982874 & 0.010642 \\ \mathrm{C} & 4.030239 & -0.352233 & 1.024703 \\ \mathrm{C} & -0.936245 & -0.133886 & 1.352524 \\ \mathrm{C} & -2.704398 & 0.658528 & -0.392451 \\ \mathrm{C} & -3.803322 & -0.086224 & 0.069866 \\ \mathrm{~N} & 5.278426 & -0.077198 & 1.405359 \\ \mathrm{C} & -5.032187 & -0.016071 & -0.541678 \\ \mathrm{C} & -0.011959 & -1.312979 & 0.989603 \\ \mathrm{H} & -0.676768 & 1.899666 & -1.435797 \\ \mathrm{H} & 1.372110 & 2.469750 & 0.134096 \\ \mathrm{H} & 3.443009 & 1.636650 & 0.691892 \\ \mathrm{H} & 0.762538 & -1.022731 & -1.004242 \\ \mathrm{H} & 1.879860 & -1.771088 & 0.075635 \\ \mathrm{H} & 3.782457 & -1.394628 & 1.029413 \\ \mathrm{H} & -1.784190 & -0.509981 & 1.907124 \\ \mathrm{H} & -0.409758 & 0.532979 & 2.025182 \\ \mathrm{H} & -2.871750 & 1.283865 & -1.253774\end{array}$




$\begin{array}{rrrr}\mathrm{H} & -3.689589 & -0.730416 & 0.921892 \\ \mathrm{H} & 5.909468 & -0.802053 & 1.666498 \\ \mathrm{H} & 5.624658 & 0.858124 & 1.436955 \\ \mathrm{H} & -5.865200 & -0.590222 & -0.182831 \\ \mathrm{H} & -5.198720 & 0.613090 & -1.397404 \\ \mathrm{H} & 0.391079 & -1.707758 & 1.916097 \\ \mathrm{H} & -0.592112 & -2.113711 & 0.540975\end{array}$

\section{TM $67^{\circ}$}

$\begin{array}{lrrr}\mathrm{C} & -0.398960 & 1.433243 & -0.585219 \\ \mathrm{C} & 0.985141 & 1.576190 & -0.077178 \\ \mathrm{C} & -1.363998 & 0.789898 & 0.160837 \\ \mathrm{C} & 1.787197 & 0.467243 & 0.086110 \\ \mathrm{C} & 3.098143 & 0.613768 & 0.624601 \\ \mathrm{C} & 1.098526 & -0.870832 & -0.119194 \\ \mathrm{C} & 3.922626 & -0.427938 & 1.007140 \\ \mathrm{C} & -0.868717 & 0.032100 & 1.381769 \\ \mathrm{C} & -2.656610 & 0.634182 & -0.399724 \\ \mathrm{C} & -3.720311 & -0.162031 & 0.098050 \\ \mathrm{~N} & 5.144382 & -0.271645 & 1.501181 \\ \mathrm{C} & -4.925433 & -0.233035 & -0.540461 \\ \mathrm{C} & 0.046975 & -1.157243 & 0.986081 \\ \mathrm{H} & -0.599166 & 1.691715 & -1.610440 \\ \mathrm{H} & 1.249145 & 2.510041 & 0.393082 \\ \mathrm{H} & 3.471067 & 1.617365 & 0.746725 \\ \mathrm{H} & 0.607649 & -0.850860 & -1.085953 \\ \mathrm{H} & 1.794085 & -1.698680 & -0.166142 \\ \mathrm{H} & 3.601781 & -1.448010 & 0.925333 \\ \mathrm{H} & -1.686040 & -0.341647 & 1.983119 \\ \mathrm{H} & -0.320926 & 0.717145 & 2.017573 \\ \mathrm{H} & -2.854020 & 1.182616 & -1.304334 \\ \mathrm{H} & -3.577506 & -0.727643 & 0.999542 \\ \mathrm{H} & 5.699999 & -1.055371 & 1.766333 \\ \mathrm{H} & 5.553751 & 0.631068 & 1.620638 \\ \mathrm{H} & -5.723682 & -0.840077 & -0.160128 \\ \mathrm{H} & -5.116094 & 0.315601 & -1.444431 \\ \mathrm{H} & 0.549195 & -1.497591 & 1.886251 \\ \mathrm{H} & -0.564762 & -1.986013 & 0.644215\end{array}$

\section{CI $73^{\circ}$}

$\begin{array}{lrrr}\mathrm{C} & -0.391487 & 1.462043 & -0.545166 \\ \mathrm{C} & 0.967266 & 1.631733 & -0.023418 \\ \mathrm{C} & -1.367839 & 0.862914 & 0.221737 \\ \mathrm{C} & 1.804008 & 0.519399 & 0.101844 \\ \mathrm{C} & 3.113635 & 0.631621 & 0.613083 \\ \mathrm{C} & 1.050109 & -0.802272 & -0.095383 \\ \mathrm{C} & 3.911000 & -0.456544 & 0.979894 \\ \mathrm{C} & -0.895360 & 0.129544 & 1.453778 \\ \mathrm{C} & -2.611932 & 0.607865 & -0.393831 \\ \mathrm{C} & -3.651560 & -0.228735 & 0.076069\end{array}$




$\begin{array}{lrrr}\mathrm{N} & 5.160462 & -0.367978 & 1.411610 \\ \mathrm{C} & -4.819869 & -0.349073 & -0.609178 \\ \mathrm{C} & 0.002159 & -1.068652 & 1.032051 \\ \mathrm{H} & -0.586719 & 1.612410 & -1.585565 \\ \mathrm{H} & 1.163952 & 2.547778 & 0.519433 \\ \mathrm{H} & 3.523522 & 1.628241 & 0.719366 \\ \mathrm{H} & 0.511191 & -0.730050 & -1.040124 \\ \mathrm{H} & 1.685675 & -1.669759 & -0.195692 \\ \mathrm{H} & 3.527625 & -1.464439 & 0.924238 \\ \mathrm{H} & -1.733911 & -0.242079 & 2.028620 \\ \mathrm{H} & -0.363819 & 0.814448 & 2.096646 \\ \mathrm{H} & -2.799717 & 1.113741 & -1.315589 \\ \mathrm{H} & -3.528443 & -0.760938 & 0.994374 \\ \mathrm{H} & 5.683732 & -1.187317 & 1.662001 \\ \mathrm{H} & 5.637768 & 0.512500 & 1.498775 \\ \mathrm{H} & -5.615082 & -0.965239 & -0.255798 \\ \mathrm{H} & -4.977419 & 0.178242 & -1.525211 \\ \mathrm{H} & 0.486144 & -1.456574 & 1.918782 \\ \mathrm{H} & -0.651656 & -1.859272 & 0.668034\end{array}$

$\mathbf{M I N}_{\mathbf{G S}}$ (photoproduct) $108^{\circ}$

$\begin{array}{lrrr}\mathrm{C} & -0.238017 & 0.840604 & -0.674735 \\ \mathrm{C} & 0.758322 & 1.235624 & 0.166246 \\ \mathrm{C} & -1.520052 & 0.424481 & -0.056716 \\ \mathrm{C} & 1.916523 & 0.332892 & 0.335522 \\ \mathrm{C} & 3.172293 & 0.769788 & 0.615271 \\ \mathrm{C} & 1.414127 & -1.104696 & 0.276761 \\ \mathrm{C} & 4.263369 & -0.097516 & 0.925864 \\ \mathrm{C} & -1.168599 & -0.477366 & 1.134167 \\ \mathrm{C} & -2.751006 & 0.727692 & -0.515105 \\ \mathrm{C} & -4.032264 & 0.273031 & 0.027089 \\ \mathrm{~N} & 5.461666 & 0.328881 & 1.149897 \\ \mathrm{C} & -5.216179 & 0.647294 & -0.490090 \\ \mathrm{C} & 0.057811 & -1.450107 & 1.002728 \\ \mathrm{H} & 0.031766 & 0.341492 & -1.592593 \\ \mathrm{H} & 0.477995 & 1.727014 & 1.085831 \\ \mathrm{H} & 3.376309 & 1.826484 & 0.593136 \\ \mathrm{H} & 1.290001 & -1.307819 & -0.783208 \\ \mathrm{H} & 2.146611 & -1.823378 & 0.629190 \\ \mathrm{H} & 4.128273 & -1.159999 & 0.987857 \\ \mathrm{H} & -2.005374 & -1.107340 & 1.408171 \\ \mathrm{H} & -0.991870 & 0.167910 & 1.990198 \\ \mathrm{H} & -2.812498 & 1.393408 & -1.360366 \\ \mathrm{H} & -4.021756 & -0.383850 & 0.878492 \\ \mathrm{H} & 6.211667 & -0.297801 & 1.368601 \\ \mathrm{H} & 5.690021 & 1.304953 & 1.112300 \\ \mathrm{H} & -6.143733 & 0.301931 & -0.073389 \\ \mathrm{H} & -5.281551 & 1.307258 & -1.337212 \\ \mathrm{H} & 0.302368 & -1.741846 & 2.018683 \\ \mathrm{H} & -0.282788 & -2.355459 & 0.512791\end{array}$


2-(4-allylidene-cycloocta-2-enylidene)-ethyliminium cation model 3

\section{Half-chair / FC}

\begin{tabular}{lrrr}
$\mathrm{C}$ & -4.044468 & 0.047986 & -2.481438 \\
$\mathrm{C}$ & -4.017883 & 0.133731 & -1.131872 \\
$\mathrm{H}$ & -5.020386 & 0.134490 & -2.927637 \\
$\mathrm{H}$ & -4.966940 & 0.191447 & -0.627231 \\
$\mathrm{C}$ & -2.849148 & 0.203962 & -0.247900 \\
$\mathrm{C}$ & -3.013814 & -0.252637 & 1.031720 \\
$\mathrm{H}$ & -3.952268 & -0.695827 & 1.317360 \\
$\mathrm{C}$ & -2.947281 & -0.211219 & -3.434831 \\
$\mathrm{C}$ & -3.065802 & 0.336327 & -4.672194 \\
$\mathrm{H}$ & -3.928428 & 0.952676 & -4.868213 \\
$\mathrm{C}$ & -2.134091 & 0.201428 & -5.789636 \\
$\mathrm{C}$ & -2.333088 & 0.800456 & -6.978733 \\
$\mathrm{H}$ & -1.253900 & -0.401085 & -5.654138 \\
$\mathrm{H}$ & -1.636907 & 0.683630 & -7.787919 \\
$\mathrm{H}$ & -3.194828 & 1.416521 & -7.166280 \\
$\mathrm{C}$ & -1.996251 & -0.213183 & 2.024828 \\
$\mathrm{~N}$ & -2.144469 & -0.704147 & 3.213088 \\
$\mathrm{H}$ & -1.040977 & 0.232466 & 1.825190 \\
$\mathrm{H}$ & -1.412386 & -0.662669 & 3.894464 \\
$\mathrm{H}$ & -2.997886 & -1.146085 & 3.498546 \\
$\mathrm{C}$ & -1.821828 & -1.169679 & -3.066775 \\
$\mathrm{H}$ & -1.624364 & -1.793325 & -3.929352 \\
$\mathrm{H}$ & -2.169891 & -1.851017 & -2.294994 \\
$\mathrm{C}$ & -1.566519 & 0.867145 & -0.725011 \\
$\mathrm{H}$ & -1.200414 & 1.506892 & 0.070431 \\
$\mathrm{H}$ & -1.810461 & 1.535534 & -1.539828 \\
$\mathrm{C}$ & -0.440936 & -0.096386 & -1.160010 \\
$\mathrm{H}$ & 0.508221 & 0.401004 & -0.988550 \\
$\mathrm{H}$ & -0.445267 & -0.979652 & -0.524686 \\
$\mathrm{C}$ & -0.496975 & -0.525790 & -2.626058 \\
$\mathrm{H}$ & -0.282304 & 0.330905 & -3.257519 \\
$\mathrm{H}$ & 0.306922 & -1.236731 & -2.789731 \\
& & & \\
\hline
\end{tabular}

\section{Boat-1}

$\begin{array}{llll}\mathrm{C} & -4.291436 & .686276 & -2.687561 \\ \mathrm{C} & -3.917470 & .864854 & -1.399170 \\ \mathrm{H} & -5.228807 & 1.144398 & -2.956292 \\ \mathrm{H} & -4.598623 & 1.414372 & -.772574 \\ \mathrm{C} & -2.686868 & .429402 & -.735480 \\ \mathrm{C} & -2.609653 & .650943 & .613368 \\ \mathrm{H} & -3.437739 & 1.115532 & 1.120074 \\ \mathrm{C} & -3.602573 & -.038593 & -3.797281 \\ \mathrm{C} & -3.280771 & .685514 & -4.895351 \\ \mathrm{H} & -3.496078 & 1.742340 & -4.874751 \\ \mathrm{C} & -2.658156 & .211755 & -6.133351 \\ \mathrm{C} & -2.359788 & 1.034853 & -7.154998 \\ \mathrm{H} & -2.439390 & -.835605 & -6.233301\end{array}$




$\begin{array}{llll}\mathrm{H} & -1.910302 & .666067 & -8.057888 \\ \mathrm{H} & -2.564951 & 2.090225 & -7.112676 \\ \mathrm{C} & -1.498258 & .283281 & 1.421387 \\ \mathrm{~N} & -1.460180 & .474738 & 2.700778 \\ \mathrm{H} & -.628933 & -.181596 & .999182 \\ \mathrm{H} & -.666880 & .206136 & 3.248966 \\ \mathrm{H} & -2.222118 & .900643 & 3.193910 \\ \mathrm{C} & -3.463845 & -1.553603 & -3.709293 \\ \mathrm{H} & -4.399031 & -1.993808 & -4.046868 \\ \mathrm{H} & -2.705353 & -1.886627 & -4.406559 \\ \mathrm{C} & -1.582195 & -.221166 & -1.539640 \\ \mathrm{H} & -1.520967 & .280861 & -2.493803 \\ \mathrm{H} & -.620167 & -.062196 & -1.066879 \\ \mathrm{C} & -1.789942 & -1.734580 & -1.747892 \\ \mathrm{H} & -.994914 & -2.083209 & -2.399910 \\ \mathrm{H} & -1.654149 & -2.244743 & -.796876 \\ \mathrm{C} & -3.145936 & -2.142843 & -2.330396 \\ \mathrm{H} & -3.150001 & -3.223634 & -2.427123 \\ \mathrm{H} & -3.940496 & -1.907786 & -1.629818\end{array}$

\section{Boat-2}

$\begin{array}{llll}\mathrm{C} & -4.247450 & .721563 & -2.706475 \\ \mathrm{C} & -3.858823 & .869997 & -1.420469 \\ \mathrm{H} & -5.153119 & 1.222378 & -3.004756 \\ \mathrm{H} & -4.531793 & 1.443819 & -.807197 \\ \mathrm{C} & -2.642408 & .413331 & -.735034 \\ \mathrm{C} & -2.575922 & .612727 & .607684 \\ \mathrm{H} & -3.414033 & 1.095139 & 1.083358 \\ \mathrm{C} & -3.570605 & .005472 & -3.819398 \\ \mathrm{C} & -3.305369 & .744422 & -4.935999 \\ \mathrm{H} & -3.525949 & 1.798186 & -4.928043 \\ \mathrm{C} & -2.742604 & .223009 & -6.137751 \\ \mathrm{~N} & -2.510994 & .952603 & -7.179605 \\ \mathrm{H} & -2.482056 & -.812897 & -6.232853 \\ \mathrm{H} & -2.112326 & .567974 & -8.013625 \\ \mathrm{H} & -2.720496 & 1.933216 & -7.194030 \\ \mathrm{C} & -1.491610 & .235693 & 1.511056 \\ \mathrm{C} & -1.530459 & .482296 & 2.833981 \\ \mathrm{H} & -.631287 & -.264237 & 1.104189 \\ \mathrm{H} & -.723327 & .194252 & 3.481031 \\ \mathrm{H} & -2.368433 & .974887 & 3.294950 \\ \mathrm{C} & -3.389465 & -1.502926 & -3.747841 \\ \mathrm{H} & -4.324445 & -1.923772 & -4.113181 \\ \mathrm{H} & -2.614482 & -1.829033 & -4.433218 \\ \mathrm{C} & -1.536309 & -.244223 & -1.535270 \\ \mathrm{H} & -1.433699 & .270573 & -2.486561 \\ \mathrm{H} & -.586125 & -.103263 & -1.037769 \\ \mathrm{C} & -1.748327 & -1.747083 & -1.769036 \\ \mathrm{H} & -.951735 & -2.118734 & -2.410139 \\ \mathrm{H} & -1.648196 & -2.267636 & -.822087 \\ \mathrm{C} & -3.104839 & -2.116903 & -2.371069\end{array}$




$$
\begin{array}{llll}
\mathrm{H} & -3.145474 & -3.193673 & -2.497826 \\
\mathrm{H} & -3.903812 & -1.864961 & -1.685089
\end{array}
$$

MEP $11^{\circ}$

$\begin{array}{lrrr}\mathrm{C} & -4.043340 & 0.046633 & -2.492712 \\ \mathrm{C} & -4.013252 & 0.131497 & -1.125680 \\ \mathrm{H} & -5.014428 & 0.157658 & -2.938624 \\ \mathrm{H} & -4.969170 & 0.182225 & -0.627618 \\ \mathrm{C} & -2.850563 & 0.208401 & -0.257783 \\ \mathrm{C} & -3.008550 & -0.253052 & 1.040224 \\ \mathrm{H} & -3.948125 & -0.694042 & 1.323964 \\ \mathrm{C} & -2.951120 & -0.214743 & -3.422289 \\ \mathrm{C} & -3.063508 & 0.339414 & -4.686972 \\ \mathrm{H} & -3.929919 & 0.957243 & -4.873589 \\ \mathrm{C} & -2.136709 & 0.199086 & -5.779697 \\ \mathrm{C} & -2.334807 & 0.802901 & -6.981830 \\ \mathrm{H} & -1.254278 & -0.404052 & -5.648584 \\ \mathrm{H} & -1.634719 & 0.682493 & -7.790682 \\ \mathrm{H} & -3.198026 & 1.420700 & -7.169330 \\ \mathrm{C} & -1.998350 & -0.210173 & 2.012563 \\ \mathrm{~N} & -2.145767 & -0.708820 & 3.224962 \\ \mathrm{H} & -1.048043 & 0.229337 & 1.817954 \\ \mathrm{H} & -1.419678 & -0.661859 & 3.894023 \\ \mathrm{H} & -2.989989 & -1.144560 & 3.503761 \\ \mathrm{C} & -1.821062 & -1.170269 & -3.065397 \\ \mathrm{H} & -1.629548 & -1.793446 & -3.931071 \\ \mathrm{H} & -2.169517 & -1.847445 & -2.291797 \\ \mathrm{C} & -1.567927 & 0.866035 & -0.726019 \\ \mathrm{H} & -1.203335 & 1.500675 & 0.073756 \\ \mathrm{H} & -1.803166 & 1.542681 & -1.536422 \\ \mathrm{C} & -0.440900 & -0.095599 & -1.159740 \\ \mathrm{H} & 0.510419 & 0.397412 & -0.989748 \\ \mathrm{H} & -0.448044 & -0.976489 & -0.522879 \\ \mathrm{C} & -0.496415 & -0.524605 & -2.626019 \\ \mathrm{H} & -0.277541 & 0.331912 & -3.257057 \\ \mathrm{H} & 0.303568 & -1.239080 & -2.792438\end{array}$

MEP $13^{\circ}$

$\begin{array}{lrrr}\mathrm{C} & -4.045557 & 0.048704 & -2.505721 \\ \mathrm{C} & -4.010063 & 0.122261 & -1.124959 \\ \mathrm{H} & -5.008764 & 0.204367 & -2.955256 \\ \mathrm{H} & -4.966169 & 0.152713 & -0.627002 \\ \mathrm{C} & -2.852186 & 0.213600 & -0.268240 \\ \mathrm{C} & -3.001698 & -0.251291 & 1.046567 \\ \mathrm{H} & -3.939496 & -0.693743 & 1.330314 \\ \mathrm{C} & -2.956570 & -0.217309 & -3.417064 \\ \mathrm{C} & -3.063318 & 0.340719 & -4.698307 \\ \mathrm{H} & -3.926667 & 0.962941 & -4.885332 \\ \mathrm{C} & -2.139114 & 0.196697 & -5.773523 \\ \mathrm{C} & -2.337626 & 0.804289 & -6.982967\end{array}$




$\begin{array}{lrrr}\mathrm{H} & -1.260443 & -0.412186 & -5.642679 \\ \mathrm{H} & -1.639513 & 0.676902 & -7.792658 \\ \mathrm{H} & -3.200036 & 1.423396 & -7.173017 \\ \mathrm{C} & -1.999580 & -0.210596 & 2.009383 \\ \mathrm{~N} & -2.146996 & -0.713746 & 3.238401 \\ \mathrm{H} & -1.050073 & 0.229070 & 1.814365 \\ \mathrm{H} & -1.422501 & -0.659848 & 3.907613 \\ \mathrm{H} & -2.989998 & -1.149773 & 3.518636 \\ \mathrm{C} & -1.821604 & -1.170187 & -3.061578 \\ \mathrm{H} & -1.634889 & -1.792463 & -3.929071 \\ \mathrm{H} & -2.167643 & -1.846764 & -2.286637 \\ \mathrm{C} & -1.569531 & 0.866496 & -0.729733 \\ \mathrm{H} & -1.207722 & 1.491317 & 0.078368 \\ \mathrm{H} & -1.794529 & 1.555410 & -1.533564 \\ \mathrm{C} & -0.440770 & -0.093470 & -1.160022 \\ \mathrm{H} & 0.511912 & 0.397397 & -0.990071 \\ \mathrm{H} & -0.451140 & -0.972708 & -0.521557 \\ \mathrm{C} & -0.495600 & -0.522391 & -2.626230 \\ \mathrm{H} & -0.275748 & 0.334396 & -3.256917 \\ \mathrm{H} & 0.302090 & -1.239193 & -2.794522\end{array}$

$\operatorname{MEP} 16^{\circ}$

$\begin{array}{lrrr}\mathrm{C} & -4.046715 & 0.057269 & -2.517883 \\ \mathrm{C} & -4.008207 & 0.105858 & -1.128059 \\ \mathrm{H} & -5.002291 & 0.253940 & -2.968310 \\ \mathrm{H} & -4.962444 & 0.116096 & -0.626786 \\ \mathrm{C} & -2.853026 & 0.214872 & -0.275682 \\ \mathrm{C} & -2.997148 & -0.248576 & 1.050468 \\ \mathrm{H} & -3.934090 & -0.694067 & 1.332566 \\ \mathrm{C} & -2.961240 & -0.217726 & -3.421056 \\ \mathrm{C} & -3.066044 & 0.338949 & -4.704504 \\ \mathrm{H} & -3.925555 & 0.965580 & -4.894129 \\ \mathrm{C} & -2.140485 & 0.193847 & -5.774986 \\ \mathrm{C} & -2.339650 & 0.803050 & -6.983181 \\ \mathrm{H} & -1.266030 & -0.420260 & -5.642768 \\ \mathrm{H} & -1.645041 & 0.670811 & -7.795235 \\ \mathrm{H} & -3.200784 & 1.423920 & -7.173113 \\ \mathrm{C} & -1.999656 & -0.213880 & 2.016762 \\ \mathrm{~N} & -2.148099 & -0.717827 & 3.250349 \\ \mathrm{H} & -1.048873 & 0.225688 & 1.822690 \\ \mathrm{H} & -1.424313 & -0.656841 & 3.921904 \\ \mathrm{H} & -2.991646 & -1.154293 & 3.532174 \\ \mathrm{C} & -1.823436 & -1.167804 & -3.058614 \\ \mathrm{H} & -1.637954 & -1.793262 & -3.924409 \\ \mathrm{H} & -2.168001 & -1.842648 & -2.282218 \\ \mathrm{C} & -1.569977 & 0.869363 & -0.733057 \\ \mathrm{H} & -1.210393 & 1.485362 & 0.083073 \\ \mathrm{H} & -1.788497 & 1.566482 & -1.531731 \\ \mathrm{C} & -0.440541 & -0.089851 & -1.161267 \\ \mathrm{H} & 0.512682 & 0.399661 & -0.990039 \\ \mathrm{H} & -0.453664 & -0.968719 & -0.522132\end{array}$




$\begin{array}{lrrr}\mathrm{C} & -0.495633 & -0.519265 & -2.626805 \\ \mathrm{H} & -0.276115 & 0.337083 & -3.257991 \\ \mathrm{H} & 0.300942 & -1.237415 & -2.795575\end{array}$

MEP $22^{\circ}$

$\begin{array}{lrrr}\mathrm{C} & -4.027095 & 0.080893 & -2.536806 \\ \mathrm{C} & -3.992607 & 0.085838 & -1.115353 \\ \mathrm{H} & -4.966148 & 0.356533 & -2.981113 \\ \mathrm{H} & -4.945647 & 0.013565 & -0.620473 \\ \mathrm{C} & -2.856239 & 0.216421 & -0.285048 \\ \mathrm{C} & -2.996082 & -0.248607 & 1.062848 \\ \mathrm{H} & -3.932407 & -0.700539 & 1.339155 \\ \mathrm{C} & -2.962746 & -0.218195 & -3.424950 \\ \mathrm{C} & -3.070008 & 0.334804 & -4.711614 \\ \mathrm{H} & -3.927744 & 0.961741 & -4.905757 \\ \mathrm{C} & -2.143973 & 0.187658 & -5.782735 \\ \mathrm{C} & -2.343597 & 0.796444 & -6.987046 \\ \mathrm{H} & -1.273652 & -0.430492 & -5.650114 \\ \mathrm{H} & -1.653113 & 0.661487 & -7.802372 \\ \mathrm{H} & -3.200843 & 1.423651 & -7.172251 \\ \mathrm{C} & -2.002734 & -0.215448 & 2.027004 \\ \mathrm{~N} & -2.151066 & -0.719911 & 3.259558 \\ \mathrm{H} & -1.047693 & 0.221907 & 1.837378 \\ \mathrm{H} & -1.428892 & -0.652132 & 3.935254 \\ \mathrm{H} & -2.995862 & -1.157041 & 3.544678 \\ \mathrm{C} & -1.825360 & -1.164084 & -3.057682 \\ \mathrm{H} & -1.641875 & -1.793854 & -3.920020 \\ \mathrm{H} & -2.170818 & -1.834275 & -2.277178 \\ \mathrm{C} & -1.569906 & 0.871617 & -0.733557 \\ \mathrm{H} & -1.217342 & 1.481064 & 0.089927 \\ \mathrm{H} & -1.784224 & 1.572631 & -1.530090 \\ \mathrm{C} & -0.441839 & -0.085752 & -1.162307 \\ \mathrm{H} & 0.510869 & 0.404210 & -0.992215 \\ \mathrm{H} & -0.452833 & -0.965377 & -0.524477 \\ \mathrm{C} & -0.498288 & -0.515414 & -2.627465 \\ \mathrm{H} & -0.279504 & 0.340664 & -3.259650 \\ \mathrm{H} & 0.298859 & -1.231755 & -2.797402\end{array}$

MEP $24^{\circ}$

$\begin{array}{lrrr}\mathrm{C} & -4.020168 & 0.089338 & -2.548364 \\ \mathrm{C} & -3.986864 & 0.079306 & -1.103767 \\ \mathrm{H} & -4.944815 & 0.415859 & -2.989583 \\ \mathrm{H} & -4.935226 & -0.053302 & -0.613467 \\ \mathrm{C} & -2.862486 & 0.218323 & -0.292233 \\ \mathrm{C} & -2.996456 & -0.247369 & 1.068378 \\ \mathrm{H} & -3.932589 & -0.701754 & 1.343030 \\ \mathrm{C} & -2.967931 & -0.221222 & -3.419337 \\ \mathrm{C} & -3.071078 & 0.333151 & -4.717360 \\ \mathrm{H} & -3.929074 & 0.957645 & -4.912081 \\ \mathrm{C} & -2.144770 & 0.187045 & -5.781920\end{array}$




$\begin{array}{lrrr}\mathrm{C} & -2.343852 & 0.796490 & -6.988370 \\ \mathrm{H} & -1.274571 & -0.430049 & -5.648193 \\ \mathrm{H} & -1.654154 & 0.661450 & -7.802807 \\ \mathrm{H} & -3.200263 & 1.423837 & -7.171658 \\ \mathrm{C} & -2.003452 & -0.215771 & 2.028846 \\ \mathrm{~N} & -2.150772 & -0.719821 & 3.259914 \\ \mathrm{H} & -1.046607 & 0.219998 & 1.837339 \\ \mathrm{H} & -1.426868 & -0.651085 & 3.936540 \\ \mathrm{H} & -2.997878 & -1.155603 & 3.547023 \\ \mathrm{C} & -1.826084 & -1.162643 & -3.055638 \\ \mathrm{H} & -1.645911 & -1.792973 & -3.918404 \\ \mathrm{H} & -2.169239 & -1.832251 & -2.273864 \\ \mathrm{C} & -1.570562 & 0.870798 & -0.735622 \\ \mathrm{H} & -1.221909 & 1.480387 & 0.089915 \\ \mathrm{H} & -1.782465 & 1.571144 & -1.533760 \\ \mathrm{C} & -0.441659 & -0.085790 & -1.162467 \\ \mathrm{H} & 0.510369 & 0.405427 & -0.992191 \\ \mathrm{H} & -0.451368 & -0.966373 & -0.525769 \\ \mathrm{C} & -0.498164 & -0.515097 & -2.627662 \\ \mathrm{H} & -0.280418 & 0.341170 & -3.259829 \\ \mathrm{H} & 0.298833 & -1.231311 & -2.798275\end{array}$

MEP $30^{\circ}$

$\begin{array}{lrrr}\mathrm{C} & -4.004926 & 0.113613 & -2.561230 \\ \mathrm{C} & -3.977402 & 0.058415 & -1.090303 \\ \mathrm{H} & -4.890060 & 0.543629 & -2.994896 \\ \mathrm{H} & -4.908772 & -0.190391 & -0.612382 \\ \mathrm{C} & -2.871774 & 0.222089 & -0.296780 \\ \mathrm{C} & -2.998335 & -0.243343 & 1.075838 \\ \mathrm{H} & -3.932734 & -0.703735 & 1.350163 \\ \mathrm{C} & -2.977932 & -0.228683 & -3.411076 \\ \mathrm{C} & -3.073021 & 0.328656 & -4.730669 \\ \mathrm{H} & -3.928240 & 0.953395 & -4.926746 \\ \mathrm{C} & -2.147615 & 0.184803 & -5.780148 \\ \mathrm{C} & -2.344179 & 0.796870 & -6.993921 \\ \mathrm{H} & -1.278584 & -0.432677 & -5.646009 \\ \mathrm{H} & -1.657056 & 0.658454 & -7.807598 \\ \mathrm{H} & -3.199497 & 1.423339 & -7.175666 \\ \mathrm{C} & -2.004642 & -0.215318 & 2.033637 \\ \mathrm{~N} & -2.150532 & -0.719761 & 3.260816 \\ \mathrm{H} & -1.046249 & 0.219928 & 1.840202 \\ \mathrm{H} & -1.425063 & -0.650564 & 3.939006 \\ \mathrm{H} & -3.000277 & -1.153280 & 3.551398 \\ \mathrm{C} & -1.829993 & -1.161679 & -3.051150 \\ \mathrm{H} & -1.652114 & -1.790754 & -3.915515 \\ \mathrm{H} & -2.166549 & -1.832875 & -2.268378 \\ \mathrm{C} & -1.574047 & 0.870970 & -0.738647 \\ \mathrm{H} & -1.227832 & 1.482139 & 0.087060 \\ \mathrm{H} & -1.781858 & 1.569692 & -1.539623 \\ \mathrm{C} & -0.442752 & -0.085372 & -1.162643 \\ \mathrm{H} & 0.507733 & 0.409261 & -0.992611\end{array}$



H $\quad-0.449164 \quad-0.967234 \quad-0.527582$
$\begin{array}{llll}\text { C } & -0.499720 & -0.513476 & -2.628115\end{array}$
$\mathrm{H} \quad-0.284888 \quad 0.343497 \quad-3.260456$
$\mathrm{H} \quad \begin{array}{llll}0.296875 & -1.229334 & -2.801677\end{array}$

\section{$\operatorname{MEP} 44^{\circ}$}

$\begin{array}{lrrr}\mathrm{C} & -3.967390 & 0.176674 & -2.559337 \\ \mathrm{C} & -3.955813 & -0.002596 & -1.088996 \\ \mathrm{H} & -4.773826 & 0.767340 & -2.957484 \\ \mathrm{H} & -4.847707 & -0.421483 & -0.653604 \\ \mathrm{C} & -2.877835 & 0.223727 & -0.283389 \\ \mathrm{C} & -3.000159 & -0.236331 & 1.086044 \\ \mathrm{H} & -3.935534 & -0.693276 & 1.365348 \\ \mathrm{C} & -2.987181 & -0.235873 & -3.416965 \\ \mathrm{C} & -3.076918 & 0.316715 & -4.746651 \\ \mathrm{H} & -3.931869 & 0.938384 & -4.948272 \\ \mathrm{C} & -2.149249 & 0.176653 & -5.788258 \\ \mathrm{C} & -2.340815 & 0.793459 & -7.003573 \\ \mathrm{H} & -1.275954 & -0.433058 & -5.648325 \\ \mathrm{H} & -1.645299 & 0.667223 & -7.811217 \\ \mathrm{H} & -3.197094 & 1.417090 & -7.185582 \\ \mathrm{C} & -2.003741 & -0.212253 & 2.040980 \\ \mathrm{~N} & -2.146952 & -0.716860 & 3.265794 \\ \mathrm{H} & -1.042238 & 0.216857 & 1.841244 \\ \mathrm{H} & -1.416219 & -0.654104 & 3.940685 \\ \mathrm{H} & -3.000063 & -1.142664 & 3.561196 \\ \mathrm{C} & -1.845473 & -1.171445 & -3.047032 \\ \mathrm{H} & -1.663752 & -1.809091 & -3.904135 \\ \mathrm{H} & -2.179460 & -1.834888 & -2.257323 \\ \mathrm{C} & -1.586718 & 0.881098 & -0.739804 \\ \mathrm{H} & -1.233892 & 1.505307 & 0.072936 \\ \mathrm{H} & -1.800773 & 1.566255 & -1.551026 \\ \mathrm{C} & -0.455080 & -0.081244 & -1.163883 \\ \mathrm{H} & 0.495048 & 0.414757 & -0.996903 \\ \mathrm{H} & -0.459087 & -0.962828 & -0.528687 \\ \mathrm{C} & -0.514854 & -0.511167 & -2.629463 \\ \mathrm{H} & -0.307546 & 0.345608 & -3.264477 \\ \mathrm{H} & 0.283496 & -1.223775 & -2.806306\end{array}$

\section{$\operatorname{MEP} 51^{\circ}$}

$\begin{array}{lrrr}\mathrm{C} & -3.944806 & 0.204811 & -2.554820 \\ \mathrm{C} & -3.940321 & -0.031989 & -1.092439 \\ \mathrm{H} & -4.719021 & 0.849840 & -2.930469 \\ \mathrm{H} & -4.815974 & -0.506182 & -0.681334 \\ \mathrm{C} & -2.879380 & 0.222685 & -0.272189 \\ \mathrm{C} & -3.000368 & -0.236112 & 1.093415 \\ \mathrm{H} & -3.935181 & -0.693693 & 1.373059 \\ \mathrm{C} & -2.990417 & -0.236689 & -3.425956 \\ \mathrm{C} & -3.077048 & 0.313321 & -4.754878 \\ \mathrm{H} & -3.929345 & 0.937596 & -4.956101\end{array}$




$\begin{array}{lrrr}\mathrm{C} & -2.149135 & 0.169668 & -5.797363 \\ \mathrm{C} & -2.336892 & 0.789879 & -7.010175 \\ \mathrm{H} & -1.276286 & -0.440256 & -5.655569 \\ \mathrm{H} & -1.638468 & 0.668701 & -7.815354 \\ \mathrm{H} & -3.193785 & 1.411185 & -7.192847 \\ \mathrm{C} & -2.002275 & -0.209805 & 2.047215 \\ \mathrm{~N} & -2.143165 & -0.714064 & 3.271182 \\ \mathrm{H} & -1.040221 & 0.218127 & 1.844028 \\ \mathrm{H} & -1.410751 & -0.654034 & 3.945123 \\ \mathrm{H} & -2.996688 & -1.137904 & 3.568419 \\ \mathrm{C} & -1.857299 & -1.180474 & -3.050370 \\ \mathrm{H} & -1.669530 & -1.822190 & -3.903673 \\ \mathrm{H} & -2.195119 & -1.839466 & -2.258155 \\ \mathrm{C} & -1.595599 & 0.890956 & -0.734955 \\ \mathrm{H} & -1.235998 & 1.518396 & 0.072200 \\ \mathrm{H} & -1.817051 & 1.572278 & -1.547846 \\ \mathrm{C} & -0.468069 & -0.075427 & -1.165019 \\ \mathrm{H} & 0.484112 & 0.418097 & -1.004202 \\ \mathrm{H} & -0.470337 & -0.955353 & -0.527698 \\ \mathrm{C} & -0.530125 & -0.511230 & -2.629917 \\ \mathrm{H} & -0.327346 & 0.344332 & -3.267869 \\ \mathrm{H} & 0.272842 & -1.219189 & -2.804535\end{array}$

MEP $57^{\circ}$

$\begin{array}{lrrr}\mathrm{C} & -3.919656 & 0.229364 & -2.550193 \\ \mathrm{C} & -3.920691 & -0.055543 & -1.096923 \\ \mathrm{H} & -4.669032 & 0.912839 & -2.906172 \\ \mathrm{H} & -4.784386 & -0.568241 & -0.708475 \\ \mathrm{C} & -2.878967 & 0.222620 & -0.259001 \\ \mathrm{C} & -3.001113 & -0.239930 & 1.101387 \\ \mathrm{H} & -3.933013 & -0.706494 & 1.375669 \\ \mathrm{C} & -2.992181 & -0.236718 & -3.436892 \\ \mathrm{C} & -3.077059 & 0.313127 & -4.763549 \\ \mathrm{H} & -3.921802 & 0.948459 & -4.960529 \\ \mathrm{C} & -2.152360 & 0.160986 & -5.809584 \\ \mathrm{C} & -2.336593 & 0.783803 & -7.020514 \\ \mathrm{H} & -1.286517 & -0.459350 & -5.670034 \\ \mathrm{H} & -1.643845 & 0.653565 & -7.828473 \\ \mathrm{H} & -3.192445 & 1.406749 & -7.203217 \\ \mathrm{C} & -2.004469 & -0.208801 & 2.056939 \\ \mathrm{~N} & -2.144097 & -0.712664 & 3.280352 \\ \mathrm{H} & -1.044933 & 0.226125 & 1.856042 \\ \mathrm{H} & -1.414049 & -0.650636 & 3.955795 \\ \mathrm{H} & -2.996188 & -1.140712 & 3.576189 \\ \mathrm{C} & -1.866905 & -1.187433 & -3.058007 \\ \mathrm{H} & -1.669879 & -1.827356 & -3.909930 \\ \mathrm{H} & -2.208115 & -1.845182 & -2.266750 \\ \mathrm{C} & -1.601630 & 0.901774 & -0.723617 \\ \mathrm{H} & -1.233958 & 1.525409 & 0.082528 \\ \mathrm{H} & -1.829130 & 1.584593 & -1.533643 \\ \mathrm{C} & -0.480292 & -0.067648 & -1.165790\end{array}$




$\begin{array}{lrrr}\mathrm{H} & 0.474513 & 0.424060 & -1.011936 \\ \mathrm{H} & -0.477410 & -0.946380 & -0.526545 \\ \mathrm{C} & -0.545552 & -0.509101 & -2.630651 \\ \mathrm{H} & -0.344795 & 0.346023 & -3.269988 \\ \mathrm{H} & 0.263795 & -1.211343 & -2.801612\end{array}$

\section{MEP $64^{\circ}$}

$\begin{array}{lrrr}\mathrm{C} & -3.883279 & 0.259173 & -2.544279 \\ \mathrm{C} & -3.891549 & -0.080642 & -1.102167 \\ \mathrm{H} & -4.595830 & 0.997322 & -2.870919 \\ \mathrm{H} & -4.736278 & -0.650316 & -0.747573 \\ \mathrm{C} & -2.876383 & 0.224398 & -0.238920 \\ \mathrm{C} & -3.000869 & -0.250578 & 1.112649 \\ \mathrm{H} & -3.929049 & -0.732592 & 1.376960 \\ \mathrm{C} & -2.992106 & -0.236708 & -3.453593 \\ \mathrm{C} & -3.075289 & 0.316905 & -4.775086 \\ \mathrm{H} & -3.908275 & 0.971007 & -4.964091 \\ \mathrm{C} & -2.160928 & 0.143733 & -5.831423 \\ \mathrm{C} & -2.341916 & 0.768117 & -7.040121 \\ \mathrm{H} & -1.306149 & -0.493938 & -5.696950 \\ \mathrm{H} & -1.657651 & 0.621986 & -7.854262 \\ \mathrm{H} & -3.190264 & 1.403828 & -7.218455 \\ \mathrm{C} & -2.010626 & -0.206421 & 2.075299 \\ \mathrm{~N} & -2.150482 & -0.711841 & 3.297223 \\ \mathrm{H} & -1.055522 & 0.242273 & 1.879496 \\ \mathrm{H} & -1.423456 & -0.643069 & 3.976281 \\ \mathrm{H} & -2.999732 & -1.151151 & 3.587652 \\ \mathrm{C} & -1.876907 & -1.196896 & -3.071719 \\ \mathrm{H} & -1.666611 & -1.833271 & -3.923986 \\ \mathrm{H} & -2.224771 & -1.856429 & -2.284757 \\ \mathrm{C} & -1.606861 & 0.917814 & -0.703644 \\ \mathrm{H} & -1.227189 & 1.532686 & 0.105039 \\ \mathrm{H} & -1.843625 & 1.608123 & -1.505703 \\ \mathrm{C} & -0.496599 & -0.055685 & -1.166736 \\ \mathrm{H} & 0.460653 & 0.433502 & -1.025006 \\ \mathrm{H} & -0.486425 & -0.932327 & -0.525341 \\ \mathrm{C} & -0.566352 & -0.503864 & -2.631461 \\ \mathrm{H} & -0.370533 & 0.350546 & -3.273718 \\ \mathrm{H} & 0.250448 & -1.197309 & -2.797358\end{array}$

MEP $67^{\circ}$

$\begin{array}{lrrr}\mathrm{C} & -3.860808 & 0.275446 & -2.539183 \\ \mathrm{C} & -3.874811 & -0.090816 & -1.106515 \\ \mathrm{H} & -4.542301 & 1.050523 & -2.845463 \\ \mathrm{H} & -4.698338 & -0.704909 & -0.776431 \\ \mathrm{C} & -2.874182 & 0.224783 & -0.226556 \\ \mathrm{C} & -3.000724 & -0.258875 & 1.118112 \\ \mathrm{H} & -3.924488 & -0.750166 & 1.378324 \\ \mathrm{C} & -2.990862 & -0.234604 & -3.462815 \\ \mathrm{C} & -3.072963 & 0.320332 & -4.781054\end{array}$




$\begin{array}{lrrr}\mathrm{H} & -3.896464 & 0.985952 & -4.967954 \\ \mathrm{C} & -2.169357 & 0.131162 & -5.846300 \\ \mathrm{C} & -2.346987 & 0.754531 & -7.053969 \\ \mathrm{H} & -1.322897 & -0.515425 & -5.715961 \\ \mathrm{H} & -1.667564 & 0.602928 & -7.868561 \\ \mathrm{H} & -3.185582 & 1.403270 & -7.230131 \\ \mathrm{C} & -2.016239 & -0.204942 & 2.088126 \\ \mathrm{~N} & -2.155505 & -0.710584 & 3.308201 \\ \mathrm{H} & -1.065433 & 0.252818 & 1.897246 \\ \mathrm{H} & -1.430739 & -0.638140 & 3.989183 \\ \mathrm{H} & -3.001308 & -1.158659 & 3.598577 \\ \mathrm{C} & -1.883741 & -1.201074 & -3.080847 \\ \mathrm{H} & -1.664292 & -1.834216 & -3.930562 \\ \mathrm{H} & -2.234938 & -1.863685 & -2.300198 \\ \mathrm{C} & -1.610711 & 0.927440 & -0.689677 \\ \mathrm{H} & -1.222529 & 1.535764 & 0.118350 \\ \mathrm{H} & -1.852573 & 1.623137 & -1.483734 \\ \mathrm{C} & -0.506431 & -0.047303 & -1.167135 \\ \mathrm{H} & 0.455005 & 0.441025 & -1.033188 \\ \mathrm{H} & -0.493051 & -0.924000 & -0.524127 \\ \mathrm{C} & -0.579401 & -0.500597 & -2.632308 \\ \mathrm{H} & -0.388032 & 0.354113 & -3.276793 \\ \mathrm{H} & 0.244393 & -1.189356 & -2.794763\end{array}$

MEP $70^{\circ}$

$\begin{array}{lrrr}\mathrm{C} & -3.837537 & 0.294377 & -2.534599 \\ \mathrm{C} & -3.856862 & -0.096829 & -1.110290 \\ \mathrm{H} & -4.500743 & 1.089957 & -2.827051 \\ \mathrm{H} & -4.669168 & -0.736048 & -0.800023 \\ \mathrm{C} & -2.869505 & 0.224198 & -0.215324 \\ \mathrm{C} & -3.001026 & -0.270249 & 1.122325 \\ \mathrm{H} & -3.921780 & -0.772847 & 1.371489 \\ \mathrm{C} & -2.986424 & -0.227570 & -3.470365 \\ \mathrm{C} & -3.071922 & 0.326568 & -4.786100 \\ \mathrm{H} & -3.886321 & 1.004619 & -4.966310 \\ \mathrm{C} & -2.183903 & 0.116209 & -5.862187 \\ \mathrm{C} & -2.363281 & 0.735861 & -7.070388 \\ \mathrm{H} & -1.347301 & -0.543859 & -5.736418 \\ \mathrm{H} & -1.694012 & 0.568522 & -7.889736 \\ \mathrm{H} & -3.192379 & 1.398076 & -7.241888 \\ \mathrm{C} & -2.026236 & -0.204657 & 2.102012 \\ \mathrm{~N} & -2.169596 & -0.712496 & 3.320071 \\ \mathrm{H} & -1.079728 & 0.265823 & 1.919487 \\ \mathrm{H} & -1.449993 & -0.633322 & 4.005863 \\ \mathrm{H} & -3.011912 & -1.172557 & 3.602386 \\ \mathrm{C} & -1.883401 & -1.198593 & -3.088775 \\ \mathrm{H} & -1.656734 & -1.826457 & -3.940530 \\ \mathrm{H} & -2.237404 & -1.864976 & -2.312204 \\ \mathrm{C} & -1.608563 & 0.934009 & -0.674199 \\ \mathrm{H} & -1.214878 & 1.534060 & 0.137318 \\ \mathrm{H} & -1.853827 & 1.635872 & -1.462267\end{array}$




$\begin{array}{lrrr}\mathrm{C} & -0.509091 & -0.040022 & -1.165198 \\ \mathrm{H} & 0.452546 & 0.448651 & -1.036213 \\ \mathrm{H} & -0.489042 & -0.917419 & -0.523644 \\ \mathrm{C} & -0.584303 & -0.492483 & -2.631549 \\ \mathrm{H} & -0.394547 & 0.362920 & -3.275358 \\ \mathrm{H} & 0.242848 & -1.176987 & -2.793731\end{array}$

MEP $75^{\circ}$

$\begin{array}{lrrr}\mathrm{C} & -3.804611 & 0.321658 & -2.528002 \\ \mathrm{C} & -3.831956 & -0.103255 & -1.115889 \\ \mathrm{H} & -4.438078 & 1.147116 & -2.799089 \\ \mathrm{H} & -4.624707 & -0.779910 & -0.835572 \\ \mathrm{C} & -2.862476 & 0.222571 & -0.199556 \\ \mathrm{C} & -3.001366 & -0.286971 & 1.127368 \\ \mathrm{H} & -3.916851 & -0.807003 & 1.361099 \\ \mathrm{C} & -2.979238 & -0.215565 & -3.480452 \\ \mathrm{C} & -3.070223 & 0.336091 & -4.793192 \\ \mathrm{H} & -3.870242 & 1.032430 & -4.964170 \\ \mathrm{C} & -2.207490 & 0.094016 & -5.885542 \\ \mathrm{C} & -2.390609 & 0.706770 & -7.094906 \\ \mathrm{H} & -1.385692 & -0.586277 & -5.767796 \\ \mathrm{H} & -1.736419 & 0.515816 & -7.922178 \\ \mathrm{H} & -3.202447 & 1.388909 & -7.259820 \\ \mathrm{C} & -2.042601 & -0.204958 & 2.122777 \\ \mathrm{~N} & -2.192740 & -0.716215 & 3.337039 \\ \mathrm{H} & -1.102249 & 0.285242 & 1.953784 \\ \mathrm{H} & -1.480957 & -0.626520 & 4.031636 \\ \mathrm{H} & -3.027997 & -1.193860 & 3.607958 \\ \mathrm{C} & -1.881613 & -1.192495 & -3.100084 \\ \mathrm{H} & -1.644788 & -1.813782 & -3.954034 \\ \mathrm{H} & -2.239885 & -1.866093 & -2.331250 \\ \mathrm{C} & -1.604446 & 0.942335 & -0.650783 \\ \mathrm{H} & -1.203176 & 1.530911 & 0.165141 \\ \mathrm{H} & -1.854564 & 1.654724 & -1.428911 \\ \mathrm{C} & -0.510650 & -0.029220 & -1.161402 \\ \mathrm{H} & 0.450571 & 0.460583 & -1.040309 \\ \mathrm{H} & -0.482839 & -0.908092 & -0.522678 \\ \mathrm{C} & -0.589476 & -0.479981 & -2.630746 \\ \mathrm{H} & -0.403729 & 0.377145 & -3.272824 \\ \mathrm{H} & 0.242029 & -1.158496 & -2.792110\end{array}$

MEP $77^{\circ}$

$\begin{array}{lrrr}\mathrm{C} & -3.782985 & 0.339297 & -2.522948 \\ \mathrm{C} & -3.816023 & -0.106525 & -1.120314 \\ \mathrm{H} & -4.397286 & 1.182739 & -2.780838 \\ \mathrm{H} & -4.595487 & -0.806188 & -0.859068 \\ \mathrm{C} & -2.857224 & 0.220685 & -0.189436 \\ \mathrm{C} & -3.002039 & -0.298415 & 1.130027 \\ \mathrm{H} & -3.913209 & -0.829687 & 1.353538 \\ \mathrm{C} & -2.973906 & -0.206271 & -3.486746\end{array}$




$\begin{array}{lrrr}\mathrm{C} & -3.068891 & 0.342680 & -4.797447 \\ \mathrm{H} & -3.859089 & 1.050640 & -4.962955 \\ \mathrm{C} & -2.223794 & 0.079185 & -5.900785 \\ \mathrm{C} & -2.408885 & 0.686287 & -7.111623 \\ \mathrm{H} & -1.412990 & -0.613971 & -5.788469 \\ \mathrm{H} & -1.767305 & 0.480170 & -7.942811 \\ \mathrm{H} & -3.210372 & 1.382803 & -7.272098 \\ \mathrm{C} & -2.053242 & -0.205076 & 2.136655 \\ \mathrm{~N} & -2.207836 & -0.718644 & 3.347919 \\ \mathrm{H} & -1.118457 & 0.297815 & 1.977118 \\ \mathrm{H} & -1.503344 & -0.622262 & 4.048375 \\ \mathrm{H} & -3.040100 & -1.209029 & 3.611396 \\ \mathrm{C} & -1.879762 & -1.187734 & -3.107156 \\ \mathrm{H} & -1.636565 & -1.803904 & -3.962557 \\ \mathrm{H} & -2.240623 & -1.865909 & -2.344024 \\ \mathrm{C} & -1.601147 & 0.947357 & -0.635546 \\ \mathrm{H} & -1.195317 & 1.528139 & 0.183654 \\ \mathrm{H} & -1.854289 & 1.666330 & -1.406343 \\ \mathrm{C} & -0.511149 & -0.022299 & -1.159005 \\ \mathrm{H} & 0.450409 & 0.468963 & -1.042375 \\ \mathrm{H} & -0.477631 & -0.902282 & -0.521996 \\ \mathrm{C} & -0.592065 & -0.471114 & -2.629484 \\ \mathrm{H} & -0.408784 & 0.387271 & -3.270849 \\ \mathrm{H} & 0.242520 & -1.146186 & -2.791303\end{array}$

\section{MEP $80^{\circ}$}

$\begin{array}{lrrr}\mathrm{C} & -3.761466 & 0.357285 & -2.518157 \\ \mathrm{C} & -3.800233 & -0.109850 & -1.124540 \\ \mathrm{H} & -4.354644 & 1.219538 & -2.762015 \\ \mathrm{H} & -4.564904 & -0.833764 & -0.883136 \\ \mathrm{C} & -2.852819 & 0.219807 & -0.178907 \\ \mathrm{C} & -3.002371 & -0.309352 & 1.132770 \\ \mathrm{H} & -3.909677 & -0.852323 & 1.346198 \\ \mathrm{C} & -2.969507 & -0.197987 & -3.493263 \\ \mathrm{C} & -3.067708 & 0.349004 & -4.802020 \\ \mathrm{H} & -3.847910 & 1.068902 & -4.961650 \\ \mathrm{C} & -2.240033 & 0.064616 & -5.916131 \\ \mathrm{C} & -2.427978 & 0.666531 & -7.127796 \\ \mathrm{H} & -1.439919 & -0.641977 & -5.809297 \\ \mathrm{H} & -1.797610 & 0.444285 & -7.964075 \\ \mathrm{H} & -3.217581 & 1.376175 & -7.284249 \\ \mathrm{C} & -2.064331 & -0.205551 & 2.150508 \\ \mathrm{~N} & -2.223960 & -0.721444 & 3.358498 \\ \mathrm{H} & -1.134231 & 0.310556 & 2.000317 \\ \mathrm{H} & -1.525227 & -0.617850 & 4.065574 \\ \mathrm{H} & -3.051472 & -1.223620 & 3.614724 \\ \mathrm{C} & -1.878249 & -1.183038 & -3.114296 \\ \mathrm{H} & -1.628222 & -1.794490 & -3.971102 \\ \mathrm{H} & -2.241576 & -1.866054 & -2.356615 \\ \mathrm{C} & -1.598081 & 0.952547 & -0.620050 \\ \mathrm{H} & -1.187330 & 1.525444 & 0.202143\end{array}$




$\begin{array}{lrrr}\mathrm{H} & -1.854081 & 1.678436 & -1.384001 \\ \mathrm{C} & -0.511589 & -0.015209 & -1.156290 \\ \mathrm{H} & 0.449923 & 0.476930 & -1.044473 \\ \mathrm{H} & -0.472904 & -0.896390 & -0.521341 \\ \mathrm{C} & -0.594798 & -0.462582 & -2.628651 \\ \mathrm{H} & -0.414196 & 0.397225 & -3.268804 \\ \mathrm{H} & 0.242779 & -1.133741 & -2.790465\end{array}$

CI $83^{\circ}$

$\begin{array}{lrrr}\mathrm{C} & -3.729896 & 0.383024 & -2.510886 \\ \mathrm{C} & -3.777195 & -0.113747 & -1.131048 \\ \mathrm{H} & -4.296966 & 1.266462 & -2.735625 \\ \mathrm{H} & -4.523256 & -0.866567 & -0.917561 \\ \mathrm{C} & -2.843590 & 0.214210 & -0.165122 \\ \mathrm{C} & -3.002957 & -0.326584 & 1.135626 \\ \mathrm{H} & -3.904089 & -0.886366 & 1.333832 \\ \mathrm{C} & -2.959560 & -0.181493 & -3.500819 \\ \mathrm{C} & -3.064872 & 0.359480 & -4.808383 \\ \mathrm{H} & -3.830430 & 1.095263 & -4.960344 \\ \mathrm{C} & -2.266100 & 0.043147 & -5.937913 \\ \mathrm{C} & -2.458065 & 0.636451 & -7.151743 \\ \mathrm{H} & -1.482783 & -0.682694 & -5.839033 \\ \mathrm{H} & -1.846324 & 0.390275 & -7.994375 \\ \mathrm{H} & -3.229433 & 1.365732 & -7.302843 \\ \mathrm{C} & -2.081382 & -0.206133 & 2.171104 \\ \mathrm{~N} & -2.248534 & -0.726109 & 3.373563 \\ \mathrm{H} & -1.159080 & 0.329425 & 2.035449 \\ \mathrm{H} & -1.559896 & -0.611805 & 4.090755 \\ \mathrm{H} & -3.069453 & -1.246665 & 3.618593 \\ \mathrm{C} & -1.874358 & -1.173570 & -3.124147 \\ \mathrm{H} & -1.615666 & -1.777456 & -3.983621 \\ \mathrm{H} & -2.241447 & -1.864552 & -2.375609 \\ \mathrm{C} & -1.592173 & 0.958171 & -0.597181 \\ \mathrm{H} & -1.175673 & 1.520265 & 0.229505 \\ \mathrm{H} & -1.852887 & 1.694184 & -1.350303 \\ \mathrm{C} & -0.510287 & -0.004972 & -1.152019 \\ \mathrm{H} & 0.450317 & 0.490037 & -1.046882 \\ \mathrm{H} & -0.463070 & -0.888053 & -0.520548 \\ \mathrm{C} & -0.596828 & -0.448968 & -2.626856 \\ \mathrm{H} & -0.420039 & 0.412953 & -3.265135 \\ \mathrm{H} & 0.244440 & -1.114983 & -2.789373\end{array}$

$\mathbf{M I N}_{\mathbf{G S}}$ (photoproduct)
$\begin{array}{llll}\text { C } & -2.889464 & 0.534940 & -2.349284\end{array}$
C $-3.028048-0.351001-1.347498$
H $-2.709265 \quad 1.571750-2.109519$
H $-3.159961-1.397845 \quad-1.571251$
C $-2.473089-0.060667-0.005907$
C $-3.216057-0.194422 \quad 1.122762$
H $-4.256261 \quad-0.458478 \quad 1.038406$ 

C $-2.520181 \quad 0.080144 \quad-3.712175$
$\begin{array}{llll}\text { C } & -3.269357 & 0.313804 & -4.808315\end{array}$
H $-4.201826 \quad 0.837458-4.671752$
$\begin{array}{llll}\text { C } & -2.968813 & -0.088147 & -6.184340\end{array}$
$\begin{array}{llll}\text { C } & -3.783116 & 0.185632 & -7.219086\end{array}$
H $-2.058637-0.629999 \quad-6.369299$
H $-3.540338-0.120246 \quad-8.219541$
H $-4.707750 \quad 0.719896 \quad-7.087134$
$\begin{array}{llll}\text { C } & -2.707786 & 0.023701 & 2.441434\end{array}$
N $-3.440755 \quad-0.061374 \quad 3.500566$
$\begin{array}{llll}\mathrm{H} & -1.677838 & 0.274188 & 2.606786\end{array}$
$\mathrm{H}-3.064931 \quad 0.095236 \quad 4.415731$
H $-4.417104-0.286432 \quad 3.449752$
C $-1.166965-0.627535-3.664148$
H $-0.696169-0.579712-4.637638$
H $-1.319765-1.685367 \quad-3.455890$
C $-1.001613 \quad 0.310745 \quad-0.101925$
$\begin{array}{llll}\mathrm{H} & -0.504942 & 0.214796 & 0.857513\end{array}$
$\begin{array}{llll}\mathrm{H} & -0.953778 & 1.365358 & -0.363998\end{array}$
C $-0.186668-0.510886 \quad-1.146713$
H $0.837990-0.464395 \quad-0.794128$
H $-0.466617-1.558920 \quad-1.083837$
C $-0.144539-0.061694 \quad-2.637714$
H $-0.130548 \quad 1.024056-2.690728$
$\begin{array}{llll}\text { H } & 0.829348 & -0.377632 & -2.996075\end{array}$

\section{$\mathrm{TS1}_{\mathrm{GS}}$}
C $\quad-3.480000 \quad 0.482561 \quad-2.491990$
C $-3.624605-0.122654-1.205291$
H $-3.771944 \quad 1.514459-2.609952$
H $-4.155908-1.059840-1.176632$
$\begin{array}{llll}\text { C } & -2.845617 & 0.251412 & -0.133026\end{array}$
C $-3.035953-0.359122 \quad 1.144952$
H $-3.930720 \quad-0.945055 \quad 1.273992$
C $-2.846901-0.158064 \quad-3.573294$
C $-3.0480640 .368305-4.832958$
H $-3.756174 \quad 1.176225 \quad-4.911660$
$\begin{array}{llll}\text { C } & -2.448471 & -0.063504 & -6.068718\end{array}$
$\begin{array}{llll}\text { C } & -2.765876 & 0.522677 & -7.245537\end{array}$
H $-1.739230 \quad-0.869004 \quad-6.052310$
$\begin{array}{llll}\mathrm{H} & -2.319497 & 0.200328 & -8.166730\end{array}$
H $-3.475352 \quad 1.328297 \quad-7.304725$
$\begin{array}{llll}\text { C } & -2.185615 & -0.237169 & 2.202644\end{array}$
$\begin{array}{llll}\mathrm{N} & -2.373743 & -0.809929 & 3.404537\end{array}$
$\mathrm{H}-1.274663 \quad 0.324295 \quad 2.124947$
H $-1.812767 \quad-0.538613 \quad 4.179362$
H $-3.241280 \quad-1.244715 \quad 3.628750$
C $-1.751125-1.142940-3.190406$
H $-1.395138-1.665389-4.067583$
H $\quad-2.130702-1.901837 \quad-2.517708$
C $-1.5849691 .015963 \quad-0.489263$ 

$\begin{array}{llll}\mathrm{H} & -1.164134 & 1.518817 & 0.370938\end{array}$
H $-1.812507 \quad 1.798687-1.209316$
C $-0.525605 \quad 0.028817-1.066956$
H $0.451295 \quad 0.475290 \quad-0.910819$
H $-0.540502-0.870828 \quad-0.459409$
$\begin{array}{llll}\text { C } & -0.543442 & -0.384319 & -2.562952\end{array}$
H $-0.342148 \quad 0.491472 \quad-3.174426$
H $0.322170 \quad-1.027923 \quad-2.681079$

\section{TS2 $_{\text {GS }}$}

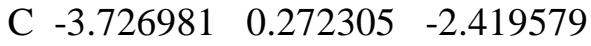
C $-3.673839-0.353571 \quad-1.097311$
H $-4.2006391 .235949-2.500066$
$\begin{array}{llll}\mathrm{H} & -4.275697 & -1.230375 & -0.921788\end{array}$
$\begin{array}{llll}\text { C } & -2.803361 & 0.083688 & -0.073584\end{array}$
$\begin{array}{llll}\text { C } & -2.984472 & -0.394464 & 1.215125\end{array}$
$\begin{array}{llll}\mathrm{H} & -3.848961 & -1.002122 & 1.420804\end{array}$
$\begin{array}{llll}\text { C } & -3.023216 & -0.243892 & -3.505479\end{array}$
C $-3.152352 \quad 0.349762 \quad-4.777021$
H $-3.909976 \quad 1.108659-4.891230$
$\begin{array}{llll}\text { C } & -2.373306 & 0.065891 & -5.947613\end{array}$
$\begin{array}{llll}\text { C } & -2.548948 & 0.714658 & -7.129705\end{array}$
H $-1.616951-0.696500 \quad-5.894748$
H $-1.955197 \quad 0.476461 \quad-7.991673$
H $-3.290867 \quad 1.484230 \quad-7.247042$
$\begin{array}{llll}\text { C } & -2.150440 & -0.078534 & 2.315689\end{array}$
$\begin{array}{llll}\mathrm{N} & -2.396394 & -0.460247 & 3.533329\end{array}$
H $-1.245918 \quad 0.481592 \quad 2.184328$
H $-1.754145-0.276200 \quad 4.278322$
H $-3.181228-1.042989 \quad 3.754051$
C $-1.919280-1.240617-3.186055$
H $-1.649940 \quad-1.805467 \quad-4.067984$
H $-2.262616 \quad-1.974501 \quad-2.463879$
C $-1.583635 \quad 0.856486 \quad-0.542522$
H $-1.122080 \quad 1.400177 \quad 0.273386$
H $-1.886852 \quad 1.606866-1.261190$
C $-0.530953-0.097374-1.178354$
$\begin{array}{llll}\text { H } & 0.427491 & 0.405769 & -1.098260\end{array}$
H $-0.451016 \quad-0.988643 \quad-0.559497$
$\begin{array}{llll}\text { C } & -0.649330 & -0.518978 & -2.664687\end{array}$
H $-0.4743390 .354380 \quad-3.285568$
H $0.194927-1.179528 \quad-2.838509$

\section{$\mathbf{T S}_{\mathrm{EX}}$}
C $\quad-3.496755 \quad 1.151461 \quad-2.565408$
C $\quad-3.421705 \quad 1.376587 \quad-1.203162$
H $\quad-4.268913 \quad 1.681194 \quad-3.098687$
H $\quad-4.100958 \quad 2.124352 \quad-0.796326$
$\begin{array}{llll}\text { C } & -2.571943 & 0.677208 & -0.227628\end{array}$
$\begin{array}{llll}\text { C } & -3.009175 & -0.522185 & 0.342586\end{array}$ 

H $\quad-3.948688-0.925939$
0.014699
C $\quad-2.681281 \quad 0.262028 \quad-3.315745$
$\begin{array}{llll}\text { C } & -3.189376 & -0.095600 & -4.589687\end{array}$
H $\quad-4.107310 \quad 0.388531 \quad-4.897095$
C $\quad-2.641055-1.022893 \quad-5.503732$
C $\quad-3.228170-1.242384 \quad-6.726395$
H $\quad-1.750637-1.574910 \quad-5.254708$
H $\quad-2.806794 \quad-1.942437 \quad-7.430377$
H $\quad-4.117920-0.713853 \quad-7.034482$
C $\quad-2.354255-1.229262 \quad 1.329916$
$\begin{array}{llll}\mathrm{N} & -2.763161 & -2.453045 & 1.796369\end{array}$
H $\quad-1.419455-0.904043 \quad 1.713711$
H $\quad-2.414337 \quad-2.724645 \quad 2.677492$
$\mathrm{H} \quad-3.707181-2.702090 \quad 1.656450$
C $\quad-1.332587 \quad-0.242721 \quad-2.813017$
$\mathrm{H} \quad-0.834862-0.763288-3.616673$
$\begin{array}{llll}\mathrm{H} & -1.504478 & -0.989711 & -2.042614\end{array}$
$\begin{array}{llll}\text { C } & -1.262555 & 1.336147 & 0.105871\end{array}$

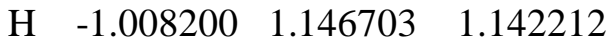
$\begin{array}{llll}\mathrm{H} & -1.361474 & 2.410897 & 0.012348\end{array}$
$\begin{array}{llll}\text { C } & -0.104552 & 0.835665 & -0.777977\end{array}$
H $\quad 0.766125 \quad 1.442975 \quad-0.559242$
H $\quad 0.145889 \quad-0.179820 \quad-0.484390$
C $\quad-0.371203 \quad 0.864453 \quad-2.288860$
$\mathrm{H} \quad-0.716395 \quad 1.851776 \quad-2.576923$
H $\quad 0.585080 \quad 0.734939 \quad-2.782470$

\section{MIN $_{\text {EX }}$}
$\begin{array}{llll}\text { C } & -3.794550 & 0.744891 & -2.543997\end{array}$
$\begin{array}{llll}\text { C } & -3.779692 & 0.907772 & -1.169477\end{array}$
H $-4.638210 \quad 1.173590 \quad-3.058391$
H $-4.619954 \quad 1.458050 \quad-0.749832$
$\begin{array}{llll}\text { C } & -2.759975 & 0.462741 & -0.204373\end{array}$
C $-3.059622 \quad-0.6312920 .614956$
H $-4.007473-1.119561 \quad 0.479874$
$\begin{array}{llll}\text { C } & -2.814867 & 0.101858 & -3.344429\end{array}$
$\begin{array}{llll}\text { C } & -3.061994 & 0.114739 & -4.738312\end{array}$
H $-3.983420 \quad 0.576714 \quad-5.066305$
C $-2.228101-0.402681-5.756924$
C $-2.588607-0.323570-7.079088$
$\mathrm{H}-1.290817-0.869235 \quad-5.506116$
H $-1.957710 \quad-0.726553 \quad-7.854896$
H $-3.5216390 .121618 \quad-7.389301$
C $-2.238869-1.161773 \quad 1.592425$
$\begin{array}{llll}\mathrm{N} & -2.529317 & -2.295665 & 2.296494\end{array}$
H $-1.281484-0.749311 \quad 1.796853$
H $-2.045538-2.443572 \quad 3.142718$
$\begin{array}{llll}\mathrm{H} & -3.460222 & -2.622707 & 2.298815\end{array}$
C $-1.560019-0.530952-2.763311$
H $-1.148261-1.230328-3.475376$
H $-1.834562-1.129280-1.901474$ 

C $-1.486121 \quad 1.258637 \quad-0.093766$
H $-1.2223591 .346686 \quad 0.954872$
$\mathrm{H}-1.656500 \quad 2.269266-0.443794$
C $-0.286528 \quad 0.650208-0.843360$
H $0.579097 \quad 1.275419 \quad-0.655548$
H $-0.063982-0.323362 \quad-0.414609$
$\begin{array}{llll}\text { C } & -0.468242 & 0.497022 & -2.355769\end{array}$
H $-0.665595 \quad 1.469433 \quad-2.796990$
H $0.4801190 .168366 \quad-2.766478$

\section{CI'}

$\begin{array}{llll}\text { C } & -3.522480 & 1.030008 & -2.373602\end{array}$

$\begin{array}{llll}\text { C } & -2.934781 & 1.672651 & -1.201187\end{array}$

H $-4.583430 \quad 1.012844 \quad-2.544213$

$\begin{array}{llll}\mathrm{H} & -2.476878 & 2.633013 & -1.367847\end{array}$

C $-2.447476 \quad 0.883133 \quad-0.158335$

$\begin{array}{llll}\text { C } & -3.095071 & -0.324176 & 0.202324\end{array}$

H $-4.039648 \quad-0.547474 \quad-0.267881$

$\begin{array}{llll}\text { C } & -2.696320 & 0.385290 & -3.273291\end{array}$

$\begin{array}{llll}\text { C } & -3.357687 & -0.296701 & -4.326817\end{array}$

H $-4.423778-0.180304-4.379319$

C $-2.775587-1.136451 \quad-5.313783$

C $-3.534175-1.749920 \quad-6.265032$

H $-1.715533-1.296965 \quad-5.313424$

H $-3.092489-2.382468-7.008113$

H $-4.598193-1.616421 \quad-6.306052$

$\begin{array}{llll}\text { C } & -2.633532 & -1.216675 & 1.155224\end{array}$

$\mathrm{N}-3.277024-2.316604 \quad 1.523556$

H $-1.701703-1.053122 \quad 1.663847$

H $-2.911373-2.920457 \quad 2.228327$

H $-4.165004 \quad-2.562927 \quad 1.137353$

C $-1.172010 \quad 0.249360 \quad-3.168438$

H $-0.802591 \quad 0.431239-4.171812$

H $-0.962959-0.799106 \quad-2.970629$

$\begin{array}{llll}\text { C } & -1.056762 & 1.258057 & 0.289318\end{array}$

$\begin{array}{llll}\mathrm{H} & -0.793053 & 0.885037 & 1.272164\end{array}$

$\begin{array}{llll}\mathrm{H} & -0.970308 & 2.338789 & 0.335017\end{array}$

C $-0.060516 \quad 0.703871 \quad-0.760470$

H $0.930275 \quad 1.040520 \quad-0.471492$

H $-0.038927 \quad-0.381586 \quad-0.694944$

$\begin{array}{llll}\text { C } & -0.269777 & 1.108877 & -2.241445\end{array}$

H $-0.527086 \quad 2.158854 \quad-2.324465$

H $0.710857 \quad 1.026983 \quad-2.699447$

Product relaxation $117^{\circ}$
$\begin{array}{llll}\text { C } & -3.145714 & 0.459484 & -2.127592\end{array}$
$\begin{array}{llll}\text { C } & -3.229968 & -0.203806 & -0.943531\end{array}$
$\begin{array}{llll}\mathrm{H} & -3.075982 & 1.533279 & -2.096833\end{array}$
$\begin{array}{llll}\mathrm{H} & -3.384299 & -1.267360 & -0.973190\end{array}$
C $\quad-2.466285 \quad 0.239701 \quad 0.231336$ 


$\begin{array}{lrrr}\mathrm{C} & -2.735375 & -0.259643 & 1.472068 \\ \mathrm{H} & -3.635189 & -0.826956 & 1.639646 \\ \mathrm{C} & -2.609027 & -0.197594 & -3.339378 \\ \mathrm{C} & -2.817292 & 0.316259 & -4.574354 \\ \mathrm{H} & -3.579884 & 1.070933 & -4.680165 \\ \mathrm{C} & -2.068441 & 0.006496 & -5.790586 \\ \mathrm{C} & -2.332110 & 0.591567 & -6.972712 \\ \mathrm{H} & -1.246723 & -0.684770 & -5.731903 \\ \mathrm{H} & -1.759276 & 0.364493 & -7.852606 \\ \mathrm{H} & -3.129925 & 1.303917 & -7.083403 \\ \mathrm{C} & -1.836043 & -0.147803 & 2.571010 \\ \mathrm{~N} & -2.080341 & -0.647337 & 3.738893 \\ \mathrm{H} & -0.885236 & 0.340871 & 2.476277 \\ \mathrm{H} & -1.433908 & -0.556638 & 4.498883 \\ \mathrm{H} & -2.936303 & -1.127982 & 3.939964 \\ \mathrm{C} & -1.452388 & -1.119769 & -2.974404 \\ \mathrm{H} & -1.097736 & -1.635356 & -3.850775 \\ \mathrm{H} & -1.770051 & -1.902849 & -2.294041 \\ \mathrm{C} & -1.174222 & 0.930108 & -0.159816 \\ \mathrm{H} & -0.687477 & 1.385563 & 0.691855 \\ \mathrm{H} & -1.392182 & 1.746266 & -0.834679 \\ \mathrm{C} & -0.176870 & -0.058563 & -0.837850 \\ \mathrm{H} & 0.809585 & 0.364888 & -0.671654 \\ \mathrm{H} & -0.181648 & -0.997730 & -0.287948 \\ \mathrm{C} & -0.248370 & -0.357961 & -2.357262 \\ \mathrm{H} & -0.093647 & 0.567908 & -2.903160 \\ \mathrm{H} & 0.636805 & -0.956785 & -2.550459\end{array}$

Product relaxation $124^{\circ}$

$\begin{array}{lrrr}\mathrm{C} & -3.017870 & 0.461258 & -2.123952 \\ \mathrm{C} & -3.109072 & -0.235023 & -0.964646 \\ \mathrm{H} & -2.930013 & 1.533695 & -2.076430 \\ \mathrm{H} & -3.275718 & -1.298246 & -1.007602 \\ \mathrm{C} & -2.413510 & 0.210822 & 0.256332 \\ \mathrm{C} & -2.782961 & -0.229877 & 1.492840 \\ \mathrm{H} & -3.710717 & -0.763602 & 1.613769 \\ \mathrm{C} & -2.547821 & -0.174002 & -3.376752 \\ \mathrm{C} & -2.871589 & 0.297202 & -4.602900 \\ \mathrm{H} & -3.664365 & 1.025553 & -4.671742 \\ \mathrm{C} & -2.218296 & -0.043296 & -5.869757 \\ \mathrm{C} & -2.484907 & 0.586118 & -7.029029 \\ \mathrm{H} & -1.469190 & -0.813509 & -5.867863 \\ \mathrm{H} & -1.969514 & 0.329146 & -7.936793 \\ \mathrm{H} & -3.227443 & 1.362477 & -7.098315 \\ \mathrm{C} & -1.949162 & -0.101284 & 2.644575 \\ \mathrm{~N} & -2.233979 & -0.614767 & 3.796424 \\ \mathrm{H} & -1.015265 & 0.423942 & 2.594511 \\ \mathrm{H} & -1.621273 & -0.513648 & 4.582712 \\ \mathrm{H} & -3.085268 & -1.122508 & 3.953060 \\ \mathrm{C} & -1.344614 & -1.060875 & -3.075557 \\ \mathrm{H} & -0.945091 & -1.474958 & -3.989620\end{array}$



H $\quad-1.642506 \quad-1.915066 \quad-2.476244$
C $\quad-1.084510 \quad 0.860651 \quad-0.079508$
$\begin{array}{llll}\mathrm{H} & -0.573635 & 1.228766 & 0.801630\end{array}$
H $\quad-1.268518 \quad 1.733454 \quad-0.692477$
$\begin{array}{llll}\text { C } & -0.140963 & -0.133480 & -0.826894\end{array}$
$\begin{array}{llll}\mathrm{H} & 0.867003 & 0.198858 & -0.603671\end{array}$
$\begin{array}{llll}\mathrm{H} & -0.233801 & -1.113945 & -0.366363\end{array}$
$\begin{array}{llll}\text { C } & -0.192271 & -0.289549 & -2.369749\end{array}$
$\begin{array}{llll}\mathrm{H} & -0.098354 & 0.690218 & -2.828843\end{array}$
$\begin{array}{llll}\mathrm{H} & 0.724733 & -0.813831 & -2.617189\end{array}$

Reagent relaxation $72^{\circ}$
C $\quad-3.739949 \quad 376940 \quad-2.518654$
C $\quad-3.769918 \quad-.094300 \quad-1.130603$
$\mathrm{H} \quad-4.469101 \quad 1.118280 \quad-2.810820$
H $\quad-4.668231 \quad-.637023 \quad-.879083$
C $-2.837725 \quad .196001 \quad-.193642$
$\begin{array}{llll}\text { C } & -2.994516 & -.329977 & 1.153768\end{array}$
$\begin{array}{llll}\mathrm{H} & -3.895890 & -.887640 & 1.349002\end{array}$

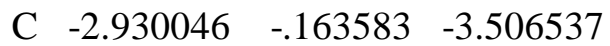
C $\quad-3.067164 \quad 365596 \quad-4.796281$
H $\quad-3.835444 \quad 1.105821 \quad-4.946017$
C $-2.273573 \quad 032143 \quad-5.944671$
C $-2.472665 \quad 627493 \quad-7.147113$
$\mathrm{H} \quad-1.487841 \quad-.690090 \quad-5.839010$
$\mathrm{H} \quad-1.854305 \quad 386168 \quad-7.990543$
H $\quad-3.242276 \quad 1.362224 \quad-7.300333$
$\begin{array}{llll}\text { C } & -2.098284 & -.203475 & 2.157757\end{array}$
$\begin{array}{llll}\mathrm{N} & -2.254288 & -.734301 & 3.397211\end{array}$
H $\quad-1.178769 \quad .333580 \quad 2.024795$
H $\quad-1.568407 \quad-.610397 \quad 4.102916$
H $\quad-3.067494 \quad-1.249054 \quad 3.644253$
C $-1.864039-1.166752 \quad-3.127665$
H $\quad-1.611465-1.778519-3.983100$
H $\quad-2.252974-1.839540 \quad-2.375285$
$\begin{array}{llll}\text { C } & -1.587777 & .954505 & -.599614\end{array}$
$\begin{array}{llll}\mathrm{H} & -1.189237 & 1.497169 & .246436\end{array}$
H $\quad-1.823235 \quad 1.716351-1.336990$
$\begin{array}{llll}\text { C } & -.507467 & -.003105 & -1.149345\end{array}$
H $\quad \begin{array}{llll}\text { 459065 } & .478908 & -1.043489\end{array}$
$\mathrm{H} \quad-.476617 \quad-.884911 \quad-.517118$
C $\quad-.590860 \quad-.444798 \quad-2.624677$
$\mathrm{H} \quad-.405204 \quad .416220 \quad-3.263302$
H $\quad .245326 \quad-1.116307 \quad-2.786982$

Reagent relaxation $60^{\circ}$
C $\quad-3.792578 \quad 336487 \quad-2.499052$
C $\quad-3.819522 \quad-.050742 \quad-1.154012$
$\mathrm{H} \quad-4.680141 \quad .828051 \quad-2.859503$
$\begin{array}{lllll}\mathrm{H} & -4.794535 & -.287670 & -.758927\end{array}$ 

$\begin{array}{llll}\text { C } & -2.805421 & .157614 & -.176963\end{array}$
C $\quad-3.008200 \quad-.321923 \quad 1.118155$
$\begin{array}{llll}\mathrm{H} & -3.907957 & -.877167 & 1.322339\end{array}$
C $-2.898942 \quad-.131615 \quad-3.508506$
$\begin{array}{llll}\text { C } & -3.063807 & .361590 & -4.780214\end{array}$
$\mathrm{H} \quad-3.841063 \quad 1.090990 \quad-4.935373$
C $\quad-2.275240 \quad .031990 \quad-5.946831$
$\begin{array}{llll}\text { C } & -2.475676 & .626064 & -7.141728\end{array}$
H $\quad-1.488038 \quad-.693248 \quad-5.850528$
$\mathrm{H} \quad-1.868623 \quad 386379 \quad-7.993910$
$\begin{array}{llll}\mathrm{H} & -3.245897 & 1.363245 & -7.285573\end{array}$
$\begin{array}{llll}\text { C } & -2.093153 & -.204228 & 2.185895\end{array}$
$\begin{array}{llll}\mathrm{N} & -2.251677 & -.725371 & 3.374676\end{array}$
$\begin{array}{llll}\mathrm{H} & -1.176659 & .338003 & 2.052654\end{array}$
$\begin{array}{llll}\mathrm{H} & -1.564656 & -.598413 & 4.089655\end{array}$

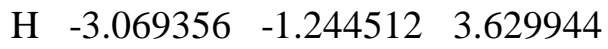
C $-1.845729-1.145416-3.123833$

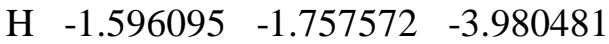
H $\quad-2.237206 \quad-1.826942 \quad-2.374801$
$\begin{array}{llll}\text { C } & -1.575503 & .933244 & -.601205\end{array}$
$\begin{array}{llll}\mathrm{H} & -1.173904 & 1.474123 & .247456\end{array}$
$\mathrm{H} \quad-1.841664 \quad 1.687133 \quad-1.329553$
C $\quad-.491016 \quad-.012419 \quad-1.152588$
H $\quad .467527 \quad .481392 \quad-1.029800$
$\begin{array}{lllll}\mathrm{H} & -.456274 & -.898206 & -.522738\end{array}$
$\begin{array}{llll}\text { C } & -.568299 & -.448346 & -2.624268\end{array}$
$\mathrm{H} \quad-.382573 \quad .414806 \quad-3.255805$
H $\quad .272748 \quad-1.116948 \quad-2.777690$ 